\title{
Simulations of stripped core-collapse supernovae in close binaries
}

\author{
Alex Rimoldi', Simon Portegies Zwart and Elena Maria Rossi
}

\begin{abstract}
We perform smoothed-particle hydrodynamical simulations of the explosion of a helium star in a close binary system, and study the effects of the explosion on the companion star as well as the effect of the presence of the companion on the supernova remnant. By simulating the mechanism of the supernova from just after core bounce until the remnant shell passes the stellar companion, we are able to separate the various phenomena leading to the final system parameters. In the final system, we measure the mass stripping and ablation from, and the additional velocity imparted to, the companion stars. Our results agree with recent work showing smaller values for these quantities compared to earlier estimates. We do find some differences, however, particularly in the velocity gained by the companion, which can be explained by the different ejecta structure that naturally results from the explosion in our simulations. These results indicate that predictions based on extrapolated Type la simulations should be revised. We also examine the structure of the supernova ejecta shell. The presence of the companion star produces a conical cavity in the expanding supernova remnant, and loss of material from the companion causes the supernova remnant to be more metal-rich on one side and more hydrogen-rich (from the companion material) around the cavity. Following the impact of the shell, we examine the state of the companion after being heated by the shock.
\end{abstract}

Keywords: supernovae; hydrodynamics; close binaries

\section{Introduction}

There is substantial evidence that most massive stars evolve in binary systems (Duquennoy and Mayor 1991; Rastegaev 2010; Sana et al. 2012). Therefore, the presence of companion star is an important consideration in the theory and observation of supernovae and supernova remnants (SNRs). In particular, while Type Ia (whitedwarf; WD) supernovae may have a companion which has deposited sufficient mass onto the WD to trigger a 'single-degenerate' explosion, many Type Ibc (stripped core-collapse) supernovae may have close companions that have been at least partly responsible for the loss of mass from the progenitor (Bersten et al. 2014; Eldridge et al. 2015; Fremling et al. 2014; Kim et al. 2015; Kuncarayakti et al. 2015).

Observational searches for supernova companions have typically focused on Type Ia explosions. Possible com-

${ }^{*}$ Correspondence: rimoldi@strw.leidenuniv.nl

Leiden Observatory, Leiden University, Niels Bohrweg 2, Leiden, 2333 CA, The Netherlands panions have been a subject of scrutiny in order to determine the frequency of the two main suspected (singledegenerate or double-degenerate) explosion channels (Maoz et al. 2014). Hydrogen enrichment from a companion has been searched for in Type Ia SNRs, but so far there has been no evidence of hydrogen lines (Leonard 2007; Lundqvist et al. 2015; Mattila et al. 2005). As noted in García-Senz et al. (2012), detection of $H_{\alpha}$ lines may be difficult due to confusion with $\mathrm{Fe}$ and Co lines due to the mostly slow $\left(<10^{3} \mathrm{~km} \mathrm{~s}^{-1}\right)$ hydrogen mixing with iron-peak elements.

The presence of a supernova companion is difficult to directly detect if they are low-mass stars at very large distances, and so far definitive evidence of close companions to any supernova progenitor, let alone those of Type Ibc, has been lacking. Tycho $\mathrm{G}$ is probably the best example of a directly imaged, suspected companion, associated with the galactic Type Ia supernova, Tycho (SN 1572; RuizLapuente et al. 2004), though recent observations put its status as a supernova companion into dispute (Kerzendorf et al. 2013; Xue and Schaefer 2015). On the other hand,

\section{空}

(c) 2016 Rimoldi et al. This article is distributed under the terms of the Creative Commons Attribution 4.0 International License (http://creativecommons.org/licenses/by/4.0/), which permits unrestricted use, distribution, and reproduction in any medium, provided you give appropriate credit to the original author(s) and the source, provide a link to the Creative Commons license, and indicate if changes were made. 
some direct searches for single-degenerate companions have ruled out giant/subgiant (evolved) stars (SN 2011fe and SNR 1006; González Hernández et al. 2012; Li et al. 2011) and even main-sequence companions (SNR 050967.5; Schaefer and Pagnotta 2012).

The presence of a companion due to increased emission, and therefore modification of the standard light curve, from the ejecta interacting with the companion has also been ruled out in observations of Type Ia supernovae (Olling et al. 2015). However, a recently observed supernova (iPTF14atg; Cao et al. 2015) does show evidence of interaction with a companion through the detection of an ultraviolet burst in the first several days.

Though much of the focus of previous work has been on Type Ia explosions, the phenomena of companion interactions with single-degenerate Type Ia ejecta has parallels with core-collapse supernovae in binaries, and therefore this scenario still provides a useful context. A similarity between Type Ia supernovae and Type Ibc supernovae is that the explosion energy in both is believed to by $E_{\mathrm{SN}} \sim$ $10^{51} \mathrm{erg} \equiv 1$ foe (Dessart et al. 2014; Smartt 2009). ${ }^{\mathrm{a}}$ Also, in single-degenerate Type Ia and binary Type Ibc explosions, main-sequence companions are typically at small orbital separations. In the former, this is simply due to the requirement for Roche-lobe overflow in the companion in order to transfer mass to the WD; in the latter, this is due to binary interactions and associated dissipative processes leading to circularised close binaries (Tauris and Takens 1998, hereafter, TT98). However, while simulations of Type Ia supernovae have placed the companion at the point of Roche-lobe overflow, the orbital separations in Type Ibc supernovae can be larger than this. Therefore, simulations of the latter are needed to test the distancedependence of results that have been extrapolated from Type Ia simulations, such as those used in Tauris (2015).

TT98 analytically investigated the consequences of a supernova in a close, circularised binary, with the goal of finding the runaway velocities of the components of a binary disrupted by a Type Ibc supernova. These predictions were based on early simulations of the effect of a supernova shell impact on a star (Fryxell and Arnett 1981) in order to determine the amount of mass lost and the change in velocity of the companion. Motivated by this problem, we perform simulations of supernovae in binary systems with properties comparable to those used in TT98.

Simulations of supernovae have been performed at many scales, ranging from hundreds of kilometres around the nascent neutron star (Janka 2012) to the impact of the ejecta shell on a companion (and the influence of the companion on the overall structure of the ejecta). The impact of Type Ia ejecta on companions has, in particular, been well studied (Liu et al. 2012; Marietta et al. 2000; Pakmor et al. 2008; Pan et al. 2012). Hirai et al. (2014) investigated the fraction of mass stripped from a giant companion star due to a effect of a core-collapse (Type II) supernova using a two-dimensional grid-based Eulerian code. Recently, Liu et al. (2015) also presented results on the consequences of a Type Ibc supernova interacting with a binary companion using smoothed-particle hydrodynamics (SPH). These studies have often focused on the companion star without following the explosion from the moment of the supernova. As a consequence, the ejecta shell is initialised artificially via analytic prescriptions near the surface of the companion, without considering its earlier evolution. In addition, the response of the binary companion and subsequent SNR evolution is analysed in these cases from a static configuration rather than placing the binary in an orbit.

For close binary orbits it is typically assumed that the binary has circularised by this point in its evolution, so that the eccentricity of the orbit can be set to zero (TT98). We follow the same assumption in this work. Moreover, despite these close separations and, therefore, short orbital periods, in theoretical work the binary period is taken to be much shorter than the timescale over which the ejected shell impacts the companion. This can be made more explicit (as in, for example, Colgate 1970) by noting that the ejecta velocity must be larger than the escape velocity of the primary star,

$$
v_{\mathrm{ej}}>v_{\mathrm{esc}}=\sqrt{\frac{2 G M_{1}}{R_{1}}}
$$

where $M_{1}$ and $R_{1}$ are the mass and radius of the primary. Since the distance, $a$, of the companion from the primary is larger than $R_{1}$, and since the orbital velocity at that distance is

$$
v_{\mathrm{orb}}=\sqrt{\frac{G M_{1}}{a}}
$$

then it must be that $v_{\mathrm{ej}}>v_{\mathrm{orb}}$. In practice, the typical ejecta velocities $\left(10^{3} \sim 10^{4} \mathrm{~km} \mathrm{~s}^{-1}\right)$ are much larger than the orbital velocities $\left(\sim 10^{2} \mathrm{~km} \mathrm{~s}^{-1}\right)$, hence the latter is typically ignored in analytic velocity calculations. However, matter in the ejecta in fact have a radially dependent velocity (approximately, in the homologous regime, $v_{\mathrm{ej}}(R, t) \propto R / t$ ). Therefore, during the late-time interactions of the lowerdensity, slower (and presumably high-metallicity) ejecta with the companion, we may no longer be justified in ignoring the orbital velocity.

Also, it is likely that the companion stars in such close orbits have been synchronised with the orbital period by tidal friction (Zahn 1977). In one of the most compact binaries we consider here, a $4 M_{\odot}$ helium star separated by $4 R_{\odot}$ from a $1 M_{\odot}$ companion, the orbital period is $4 \times 10^{4} \mathrm{~s}$, which is still much longer than the timescale of the interaction of the supernova ejecta $\left(\lesssim 2 \times 10^{3} \mathrm{~s}\right)$. With synchronisation, the surface of a star at $\sim 1 R_{\odot}$ would therefore rotate 
at $2 \pi R_{\odot} /\left(4 \times 10^{4} \mathrm{~s}\right) \approx 100 \mathrm{~km} \mathrm{~s}^{-1}$. As this is also orders of magnitude smaller than the ejecta velocity, we do not expect rotation to induce any substantial asymmetries during the shell interaction and do not consider it here. ${ }^{\mathrm{b}}$

An additional important factor in the dynamics of supernovae in binaries is a possible kick imparted to the newly formed neutron star. This is likely due to a 'gravitational tugboat' effect from asymmetry in the ejecta surrounding the neutron star after the core bounce, and perhaps also high magnetic fields and the asymmetric emission of neutrinos from the proto-neutron star (Kusenko and Segrè 1996; Maruyama et al. 2011; Scheck et al. 2004, 2006; Wongwathanarat et al. 2013). For ultra-stripped supernova progenitors, which have very small ejecta masses, the shock expands very rapidly and the tugboat effect on the neutron star has been shown to be minimal (Suwa et al. 2015). For the range of hydrodynamic simulations in this paper we do not apply any additional kick to the neutron star.

To study this problem hydrodynamically, we simulate a supernova in an orbiting binary from just after the moment of core bounce in the supernova. To this end, we first generate stellar structure models of the binary components using a one-dimensional stellar evolution code, where we strip an evolved massive progenitor of the majority of its envelope. We then convert these stellar structures to three-dimensional stars in an SPH code, and run simulations from the moment of the supernova. We vary the mass of the primary star as well as the orbital separation independently. In particular, we are interested in investigating the dependence of the companion's removed mass and impact velocity (the radial component in velocity of the companion induced by the impact of the shell) on the initial orbital separation of the binary. We describe our numerical method in more detail in the following section.

\section{Method}

Throughout this work we use the Astrophysical Multipurpose Software Environment ${ }^{\mathrm{c}}$ (AMUSE; Pelupessy et al. 2013; Portegies Zwart et al. 2009, 2013) to perform our simulations. We first outline the technique used to generate the stellar models in our binary systems (Section 2.1). We then describe the set up of the initial hydrodynamical models from the stellar structure (Section 2.2). Finally, we describe the simulation of the supernova in the binary, with some discussion on the initial convergence tests that were performed (Section 2.3).

\subsection{Stellar models}

In order to generate an SPH realisation of the binary, we require a stellar evolution code that can return the internal structure of the star. Two evolution codes in AMUSE fit this criterion: MESA (Paxton et al. 2011) and EVtwin (Eggleton 1971, 2006). We chose MESA to evolve the models to their final stellar structure, motivated by difficulties in previous work in using EVtwin to obtain solutions past the carbon flash in more massive stars (de Vries et al. 2014).

Due to interactions with the binary companion (and potentially also through stellar winds), much of the mass of the primary star is lost over its lifetime, resulting the helium star progenitor of a Type Ibc supernova (for some observationally-motivated examples, see Kim et al. 2015). To obtain an estimated lifetime of the progenitor, we first use SSE, which is a fast predictor of stellar properties based on parametrised stellar evolution tracks (Hurley et al. 2000). With the intent of generating $3 M_{\odot}$ and $4 M_{\odot}$ Helium-star progenitors, we begin with a $12.9 M_{\odot}$ and $16.0 M_{\odot}$ zero-age models with metallicity $Z=0.02$, ${ }^{\mathrm{d}}$ which are predicted by SSE to end with the required helium core masses.

We do not model or speculate on the specific mechanisms of the mass loss from the primary star, but instead apply a constant mass loss (removed from the outer mass shells of the MESA structure model) until the final helium star mass is reached. Because the lifetime in SSE may be an overprediction compared to the actual lifetime reached in MESA, we apply this mass loss between 80 and 90 per cent of the predicted SSE lifetime so as to not reach the end of the MESA evolution before all of the required mass is stripped. The stellar evolution is then continued until the final lifetime found in MESA.

For our helium star models, the very final stage of evolution involves a rapid expansion of the remaining, tenuous envelope. Due to interaction with the close binary companion, this small amount of material in the envelope is in fact expected to be lost from the system. For our progenitors, we use models just prior to this stage, at which the outer radius of the helium star is still compact (this corresponds to an age of $19 \mathrm{Myr}$ and $14 \mathrm{Myr}$ for the $3 M_{\odot}$ and $4 M_{\odot}$ helium stars, respectively). For consistency, we evolve our $\left(Z=0.02, M_{2}=1 M_{\odot}\right)$ companion star to the same age as that used for the primary star. This means the companion is still very early on the main sequence, and therefore the effect of this small duration of stellar evolution on the structure and composition of the companion is negligible.

\subsection{Hydrodynamical model set-up}

We model the hydrodynamics of the supernova using the SPH code Gadget-2 (Springel 2005), running in the AMUSE framework. The SPH formalism has been shown to be effective in three-dimensional simulations of stellar phenomena (for example, Pakmor et al. 2012). One reason is that computational resources are not expended on regions of vacuum or negligible density (for example, Church et al. 2009; Lai et al. 1993), which constitute a significant fraction of the simulation volume in the current problem. Modelling a binary in a vacuum is easily handled in SPH, 
without the need for (low density) background fields in grid codes, which can exhibit artificial shocks from motions of other bodies within this background.

The Lagrangian nature of SPH describes advection naturally, without suffering from complications of numerical diffusion found in Eulerian codes, and we do not have to restrict the simulation to a fixed volume, which is useful in the present problem of a rapidly expanding shell of gas. A benefit to running the simulation in three dimensions is the absence of any boundary effects, which can produce on-axis artefacts (Marietta et al. 2000) or preferential wave numbers in the formation of instabilities (Warren and Blondin 2013). As with all hydrodynamical codes, the SPH method also has its drawbacks, and some of these are discussed further in the context of our convergence studies in Section 2.5.

The stellar models created in MESA are converted into SPH particles using the star_to_sph routine in AMUSE, in a similar method to that outlined in de Vries et al. (2014). The routine first extracts the one-dimensional hydrostatic structure of the star, represented as a function of mass coordinates, from the data generated by the stellar evolution code. The SPH particles are initialised in a homogeneous sphere constructed from a face-centred cubic lattice, and the radial positions of the particles are then adjusted so as to match the density profile from the evolution code. ${ }^{\mathrm{e}}$ The internal energies of the particles are then assigned from the temperature (and mean molecular weight) distribution from the stellar evolution code. We use equal-mass particles throughout these simulations (unequal-mass particles can cause additional complications such as spurious mixing; Rasio and Lombardi 1999).

The primary star is configured with a purely gravitational core particle of $1.4 M_{\odot}$ to model the neutron star. The softening length $\epsilon$ is chosen to be equal to the smoothing length, such that, due to the compact support of the cubic spline, the smoothing kernel reaches zero at $2.8 \epsilon$. This equality is also maintained for the SPH particles to preserve equal resolution of the gravitational and pressure forces. The zero-kinetic-energy models are relaxed over 2.5 dynamical timescales of the gas using critical damping on the velocities of the particles, where at each step the magnitude of damping is reduced so that in the final step no constraint on the velocity is imposed (for a similar approach, see de Vries et al. 2014). This is required due to effects of mapping the one-dimensional stellar structure on to the particle grid, and differences in physics between the codes, such as the value of the adiabatic exponent (which, in the SPH code, is a fixed value of $\gamma=5 / 3$ ).

We show the one-dimensional stellar structures as well as the relaxed SPH particle densities in Figure 1. Note that for the primary stars, the density according to the SPH gas particles levels off towards the centre compared with the

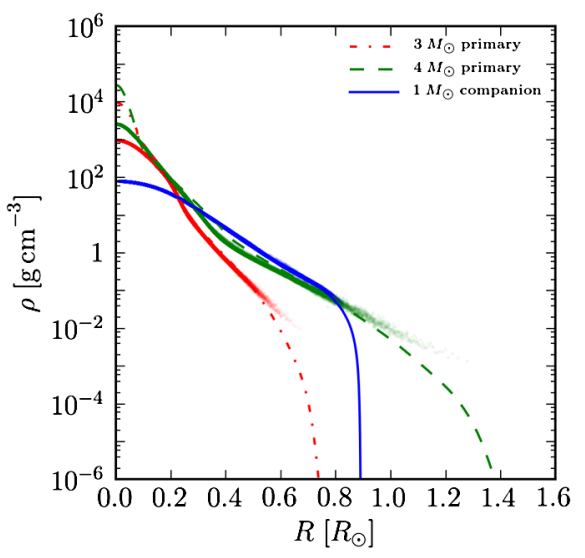

Figure 1 Comparison of the internal stellar structures of the $3 M_{\odot}$ and $4 M_{\odot}$ primary stars, as well as the $1 M_{\odot}$ companion. Lines correspond to the one-dimensional models given by MESA, where the solid line is the companion star, the dot-dashed line is the $3 M_{\odot}$ primary and the dashed line is the $4 M_{\odot}$ primary. Overlaid in the same colour are points representing the densities calculated at the location of each SPH particle in the relaxed SPH models constructed from the MESA models (which excludes the neutron star at the origin).

MESA profiles, as the central density in this region is dominated by the single core particle (which is excluded from the densities in the plot).

We set up the binary models at different orbital separations, $a$, where the minimum separation is chosen to be greater than the limit of Roche-lobe overflow (RLOF) of the companion star, given by the Eggleton (1983) relation,

$$
a_{\mathrm{RLOF}}=\frac{0.6 q^{2 / 3}+\ln \left(1+q^{1 / 3}\right)}{0.49 q^{2 / 3}} R_{2},
$$

where $R_{2}$ is the companion radius and $q$ is the binary mass ratio $M_{2} / M_{1}$. Once both stars have been constructed in the SPH code, orbital velocities are determined for a circular orbit at the specified separation and applied to each star.

\subsection{Simulation of the supernova explosion}

The supernova is initiated using the 'thermal bomb' technique (Hirai et al. 2014; Young and Fryer 2007), which assumes the core bounce has just occurred, at which moment we inject energy into a shell of particles around the neutron star. As discussed in Young and Fryer (2007), thermal bomb approaches (along with alternative, pistondriven shocks) are not intended to embody the physical mechanism that drives the supernova. Indeed, the actual processes by which the energy gain occurs near the proto-neutron star are still not fully understood, though recent observations and insights from simulations have shed some light on the role of instabilities, asymmetries and jets in driving this process (Bruenn et al. 2013; Couch and O'Connor 2014; Couch and Ott 2015; Hanke et al. 
2013; Janka 2012; Lopez et al. 2013; Milisavljevic et al. 2013).

The boundary of energy injection is specified by radius (which is equivalent to a fixed enclosed mass) instead of particle number. This allows scaling of the problem over a range of SPH particle numbers while keeping fixed the mass fraction that receives the supernova energy. The total thermal energy (a canonical $10^{51} \mathrm{erg} \equiv 1 \mathrm{foe}$ ) is distributed evenly amongst these $N_{\mathrm{SN}}$ particles, so that the specific internal energy per particle is increased by (1 foe) $/\left(N_{\mathrm{SN}} m_{\mathrm{SPH}}\right)$.

We found that a careful investigation of the effect of the injection radius was necessary. Too small a radius (and therefore $N_{\mathrm{SN}}$ ) results in large asymmetries in the shock front that grow from intrinsic small-scale asymmetries in the initial particle distribution. On the other hand, too large a radius results in the internal energy of the supernova being distributed amongst a large number of particles, lowering the specific internal energy and therefore reducing the overall temperature in the region and weakening the shock. We found that, for the helium star models used here, injecting the supernova energy into a region $R_{\mathrm{SN}} \lesssim 0.05 R_{\odot}$ generates a sufficiently spherical shock while still keeping $N_{\mathrm{SN}}$ sufficiently small.

To check the strength of the resulting shock, we calculated the Mach number at various stages through one of our supernovae (the $3 M_{\odot}$ primary). After $2 \mathrm{~s}$, in the hightemperature core the Mach number is $\sim 3$, but quickly grows as the shock traverses down the temperature gradient of the star (where the sound speed is lower). The Mach number exceeds 10 by shock breakout. Increasing the radius of energy injection would reduce the initial energy density and therefore the initial Mach number. However, provided the Mach number remains high (as seen in our simulations), the strong-shock conditions are upheld.

\subsection{Measured parameters}

With the results of these simulations it is possible to predict the final velocities (formally, at infinity) of the runaway components of supernova-disrupted binaries. For masses $m$ relative to the neutron star mass (i.e. $m \equiv M / M_{\mathrm{NS}}$ ), TT98 calculate these values in terms of the following initial parameters:

- $a$ : the pre-supernova binary orbital separation

- $v$ : the pre-supernova relative orbital velocities

- $w$ : the magnitude of the kick applied to the NS

- $\theta$ and $\phi$ : the spherical polar angles of the NS kick vector with respect to the ' $x$ '-axis aligned along the NS orbital velocity vector at the moment of the kick

- $v_{\mathrm{im}}$ : the magnitude of the radial velocity component imparted to the companion due to the impact of the supernova shell (we refer to this as the 'impact velocity')

- $v_{\mathrm{ej}}$ : the magnitude of the radial velocity of the ejecta shell
- $m_{2}, m_{2 f}$ and $m_{\text {shell }}$ : the initial mass of the companion, the final mass of the companion after mass loss, and the mass of material in the shell, respectively (all relative to the neutron star mass)

In the original work of Wheeler et al. (1975), during the supernova shell passage over the companion star, the mass removed from the companion is parametrised by the fraction of companion radius $x=R / R_{2}$ as a function of the angle around the star. Above some critical fraction of the companion radius, $x_{\text {crit }}$, a fraction $F_{\text {strip }}$ of the mass is stripped by the shell impact, and below it a fraction $F_{\text {ablate }}$ of mass is ablated. The values of $F_{\text {strip }}$ and $F_{\text {ablate }}$ are calculated in Wheeler et al. (1975) using a polytropic star of in$\operatorname{dex} n=3$. The predictions in TT98 are based on the work of Wheeler et al. (1975) as well as mass-loss estimates from simulations of a planar slab of material hitting a star Fryxell and Arnett (1981), which have a low resolution compared with modern simulations. These results also need a corrective factor due to the shell in reality having some curvature. Higher resolution simulations, such as those presented here, provide a test of these earlier estimates, which are one of the sources of uncertainty in the results of TT98.

The magnitude of the radial impact velocity imparted to the companion by the shell, $v_{\mathrm{im}}$, is theoretically determined to be

$$
v_{\mathrm{im}}=\eta v_{\mathrm{ej}}\left(\frac{R_{2}}{2 a}\right)^{2} \frac{M_{\mathrm{ej}}}{M_{2}} x_{\mathrm{crit}}^{2} \frac{1+\ln \left(2 v_{\mathrm{ej}} / v_{\mathrm{esc}}\right)}{1-F^{*}} .
$$

Here, we use the expression from Wheeler et al. (1975) in the form adopted by Tauris (2015), which applies the substitution $\left(F_{\text {strip }}+F_{\text {ablate }}\right)=F \rightarrow F^{*}=\left(F_{\text {strip }}+F_{\text {ablate }}\right)^{\alpha}$ for some $\alpha$, as well as a free parameter $\eta$ to account for the fact that this tends to over-predict the value of $v_{\mathrm{im}}$. Effectively, $\eta$ represents the final change in momentum, $\Delta p$, of the companion as a fraction of the incident momentum in the shell. As noted in Wheeler et al. (1975), corrections must be applied to this formula as it neglects the presence of a rarefaction wave back through the ejecta, geometrical effects of curvature in the shell (more important for small $a$ ), inhomogeneities in the ejecta and radiative losses behind the shock. Further phenomena can also modify the final impact velocity, such as the deformation of the companion by the shock passage (altering its cross-sectional area), the formation of a bow shock in the ejecta (during which time the flow deflects around the companion star), and shock convergence on the far side of the star (causing the asymmetric emission of material from this side of the star).

In our simulations, the two main measurements we make are, therefore, the mass loss from and impact velocity imparted to the companion, as a function of orbital separation. We measure the removed mass by calculating the specific energy for each particle,

$$
e_{\mathrm{tot}}=e_{\mathrm{kin}}+e_{\mathrm{therm}}+e_{\mathrm{pot}}=\frac{1}{2} v^{2}+u-\phi,
$$


where $v$ is the particle velocity, $u$ is its specific internal energy and $\phi$ is the gravitational potential at its position. Bound particles have a negative value of $e_{\text {tot }}$. The amount of bound mass in the companion is time-dependent over the course of the simulation due to energy exchange between particles. The stabilisation of mass bound in the companion determines the end of our simulation, which occurs within 10 dynamical timescales of the companion star $\left(\sim 2 \times 10^{4} \mathrm{~s}\right)$. The mass-loss results will be discussed in Section 3.2. Measurements of the radial impact velocity that is gained by the companion will be discussed in Section 3.3.

\subsection{Convergence test}

One feature of SPH that requires caution is that the resolution is dependent on the local density, and therefore the method loses resolution in the lower-density, uppermost layers of the stars in our simulations. In the current problem, the mass stripped by the secondary is from these same layers. Therefore, a good test for the resolution of the simulations is to look for convergence in the quantity of mass stripped from the companion.

During the supernova, Richtmeyer-Meshkov (the impulsive form of Rayleigh-Taylor) instabilities (RMI) are expected to be present, which have been found to appear once reverse shocks form at the interfaces between discontinuities in the density gradient (Kane et al. 1999). Such discontinuities are present in Type Ibc progenitors at the interface between the carbon-oxygen boundary in the core and, if any substantial fraction of hydrogen remains in the envelope, also at the helium-hydrogen boundary. However, these discontinuities tend to be smoothed during the conversion from the one-dimensional stellar model and subsequent relaxation of the SPH particles. Proper treatment of the RMI requires a prescription of artificial conductivity that is not included in the current SPH codes in AMUSE. This instability is expected to be a significant factor in the mixing of stellar material early in the evolution of SNRs, and so any evaluation of the fate of the composition of the supernova ejecta must take this into account.

Unless the growth of RMI is explicitly seeded by some structure at the density interface, these instabilities will grow from perturbations at the numerical level of the simulation and may not, in such cases, grow substantially (Kane et al. 1999). Therefore, there is the potential for instabilities to be dependent on numerical effects such as the resolution of the simulation. Additionally, during the stripping of mass from the companion star, the initial deceleration of the shell impacting the companion may be Rayleigh-Taylor unstable, but also that the subsequent flow of the shell over the surface of the star may induce some shearing (Kelvin-Helmholtz) instabilities (KHI).

Due to the smoothing of discontinuities after relaxation of the SPH models, a lack of artificial conductivity ${ }^{\mathrm{f}}$ in

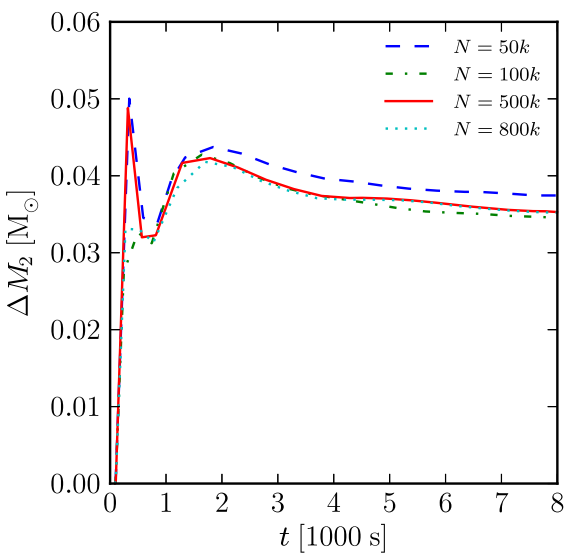

Figure 2 Results for a convergence study using the amount of mass stripped from the companion. The primary star mass was $3 M_{\odot}$ and the orbital separation was $4 R_{\odot}$ in all cases. The number of $\mathrm{SPH}$ particles used in each run is given in the legend.

Gadget -2 and the only perturbations being from noise in our SPH distribution, we expect that instabilities will not be fully captured in our simulations. As a result, we expect that the influence of instabilities on our results should also be reduced.

Figure 2 shows a test of varying the SPH particle number, $N$, based on the amount of mass lost from the companion star (evaluated using Equation 5). For low $N$, there is noticeable noise in the bound mass determination over time, but for $N \geq 10^{5}$ particles, this is no longer appreciable. As shown in Figure 2, we did not find any substantial difference in the results increasing $N$ from $5 \times 10^{5}$ to $8 \times 10^{5}$. Accounting for this, as well as available computational resources, our simulations were run with $5 \times 10^{5}$ particles.

\section{Results}

After reviewing the initial conditions used for our simulations, we examine the early stages of the supernova (Section 3.1). We then investigate the magnitude of mass lost from the companion as a function of the orbital separation (Section 3.2), as well as the velocity imparted to the companion and the fraction of imparted momentum compared to the incident shell (Section 3.3). Next, we examine the newly formed SNR for asymmetries in morphology and metallicity (Section 3.4). Finally, we consider the subsequent evolution of a star altered by a supernova shell impact (Section 3.5).

Table 1 shows the initial conditions used in our simulations. The choice of primary and companion masses is motivated by the binary parameters used in TT 98 and Tauris (2015), while the minimum orbital separations are chosen to be outside the RLOF value (Equation 3). The final two columns show the effects on the companion due to the shell impact, discussed in more detail in the remainder of this section. 
Table 1 Simulation initial conditions and main results

\begin{tabular}{lllll}
\hline $\boldsymbol{M}_{\mathbf{1}}\left(\boldsymbol{M}_{\odot}\right)$ & $\boldsymbol{M}_{\mathbf{e j}}\left(\boldsymbol{M}_{\odot}\right)$ & $\boldsymbol{a}\left(\boldsymbol{R}_{\odot}\right)$ & $\boldsymbol{\Delta} \boldsymbol{M}_{\mathbf{2}}\left(\boldsymbol{M}_{\odot}\right)$ & $\boldsymbol{v}_{\mathbf{i m}}\left(\mathbf{k m ~ s}^{-\mathbf{1}}\right)$ \\
\hline 4.0 & 2.6 & 4.5 & 0.021 & 78 \\
4.0 & 2.6 & 5.5 & 0.013 & 57 \\
4.0 & 2.6 & 6.5 & 0.0096 & 47 \\
3.0 & 1.6 & 4.0 & 0.037 & 83 \\
3.0 & 1.6 & 5.0 & 0.020 & 60 \\
3.0 & 1.6 & 6.0 & 0.013 & 48
\end{tabular}

The first three columns indicate the initial conditions, where $M_{1}$ is the mass of the primary (helium star) and before the supernova, $M_{\mathrm{ej}}$ is the total ejecta mass, and $a$ is the initial orbital separation. The last two columns are the amount of mass stripped from the companion star and the (magnitude of the) impact velocity.

\subsection{Shock breakout}

By approximately $20 \mathrm{~s}$ after the supernova is initiated, the forward shock has broken out of the surface of the helium star, during which time the fraction of SPH particles bound to the $1.4 M_{\odot}$ neutron star drops smoothly to almost zero. We find at late times that there is some fall-back of a small amount of material, which remains bound to the neutron star. As we do not model here the complexities of the magnetic field of the new neutron star or any form of pulsar wind, it is possible that other mechanisms later expel some or all of the residual bound gas.

In Figure 3, it can be seen that there is a rapid conversion of energy from internal (thermal) energy from the moment of explosion to kinetic and potential energy as the shock passes through the star and the subsequent shell expands. By approximately $100 \mathrm{~s}$ following the supernova explosion, very little of the original thermal energy remains in the gas as it has been almost entirely converted into kinetic energy in the expanding shell.

Figure 4 shows the changes in density through the $3 M_{\odot}$ helium star from shortly after core bounce until after the forward shock has reached the outer layers of the star. A lower density cavity with a very shallow gradient is seen to lag behind the expanding ejecta shell. After the shock has reached the surface (24 s), the expansion proceeds towards the companion in a self-similar manner - the variation in gradient is maintained over time, although the overall magnitude of the density drops during the expansion. We investigate the density and velocity distributions within the ejecta in more detail in Section 3.3.

\subsection{Impact and mass loss from the companion}

We now consider the phenomena occurring during the supernova shell impact on the companion, as well as the removal of mass during this interaction. The passing shell first strips material from the outer layers of the companion. The compression of the companion along the direction of motion of the shock causes heating of the stellar material, which results in a subsequent mass loss through ablation. This ablation of material is found to be a slower form of mass loss than the initial stripping phase.

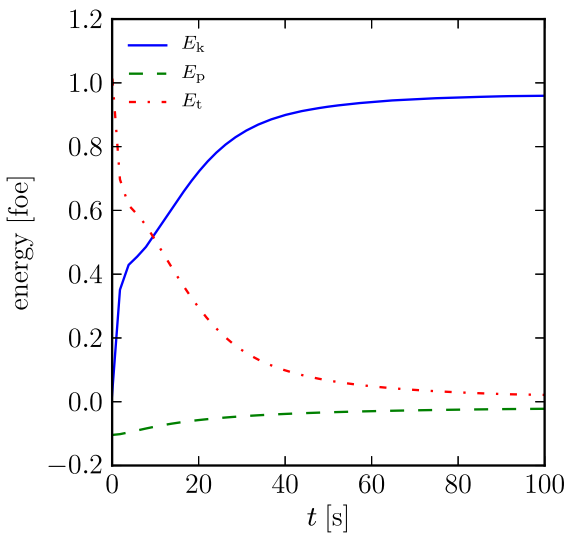

Figure 3 Distribution of total energy in the gas, in units of foe $\left(10^{51}\right.$ ergs), as a function of time following the supernova event. The energy is broken down into kinetic $\left(E_{\mathrm{k}}\right)$, potential $\left(E_{\mathrm{p}}\right)$ and internal (thermal) $\left(E_{\mathrm{t}}\right)$. This example corresponds to a primary of mass $4 M_{\odot}$ and an orbital separation of $4.5 R_{\odot}$.

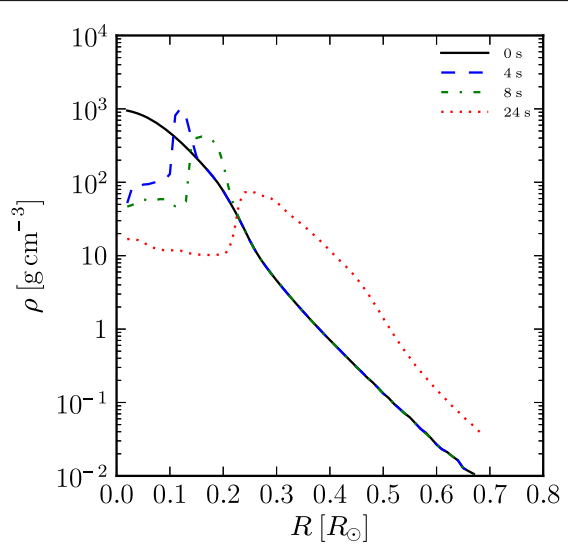

Figure 4 The radial density structure of SPH particles (excluding the core particle) at $4 \mathrm{~s}, 8 \mathrm{~s}$ and $24 \mathrm{~s}$ following a supernova in a $3.0 M_{\odot}$ primary.

The passage of the shock through the companion can be seen in the density slices of Figure 5 . The black vectors in this figure show the velocities for a random sample of all the SPH particles that were originally in the companion which have subsequently become unbound. These vectors have had the orbital velocity vector of the companion subtracted, and they are then projected onto the orbital plane. Because each SPH particle has the same mass, these vectors also indicate the relative momentum of the unbound particles.

Aside from the mass stripping from the sides of the star as predicted in Wheeler et al. (1975), there is also mass loss from the far side after the shock has passed through the star. Panel (b) of Figure 5 shows that, once the shock passes through the centre of the companion, it converges at the far 


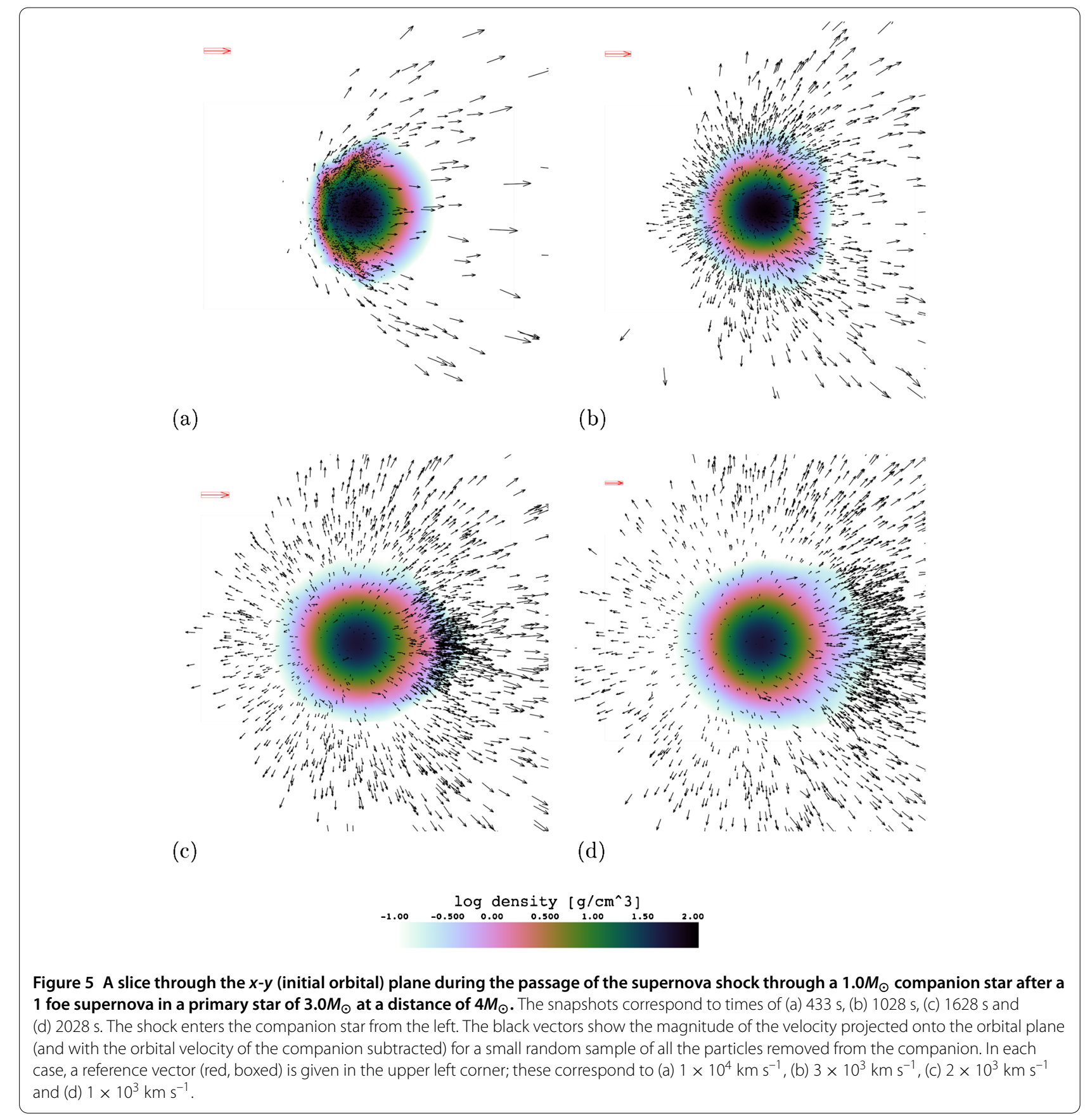

side of the star as it accelerates down the density gradient (similar shock convergence is seen around other spherically symmetric density gradients, such as in Rimoldi et al. 2015). This increases the local pressure on this axis, resulting in expulsion of material from the far side of the star (panels (c) and (d)) and can counter the effect of the outward kick imparted by the incident shell of material (see also Marietta et al. 2000).

In the last panel of Figure 5, the central density of the companion has dropped and it has noticeably expanded from the shock heating. During this later stage (final three panels) ablation occurs for material which has been heated to the point where the thermal energy is greater than the binding energy. Due to the shock heating, the companion becomes extended, similar to a pre-main sequence star (though its internal structure will differ from a pre-main sequence star), and its luminosity is expected to increase temporarily as it reverts to thermal equilibrium (Marietta et al. 2000; see also Section 3.5). Finally, we find a quadrupole oscillation of the companion that is induced 


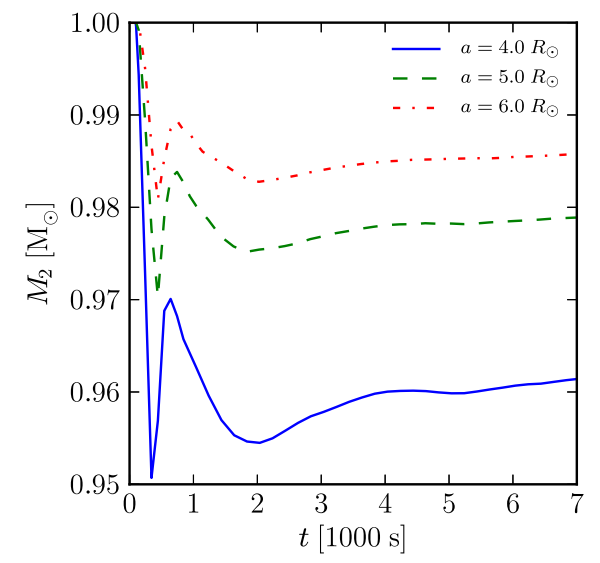

Figure 6 Mass bound to the companion using Equation 5 as a function of time since the supernova explosion for a primary helium star of $3.0 M_{\odot}$ and a range of orbital separations.

by the distortion from compression due to the shock. This ringing subsides after about one dynamical timescale of the companion star.

Figure 6 shows an example of the variation in companion mass due to the shell impact. The stripping of mass by the passing shell causes a rapid mass loss in the initial phase. There is then a brief increase in the bound mass, which has also been seen in past simulations (Liu et al. 2015; Pakmor et al. 2008; Pan et al. 2012). One possible cause of this is the formation of a reverse shock in the ejecta, which slows material with respect to the companion and increases the amount of bound mass (Pan et al. 2012). A more gradual mass loss then ensues due to the later ablation of shockheated material. The proportion of mass lost drops rapidly even by moderate orbital separations.

In Figure 7, we show the amount of mass lost from the companion (as a fraction of its initial mass) as a function of orbital separation. The lost mass is found by subtracting the final bound mass at the last snapshot of each simulation (which occurs at $2 \times 10^{4} \mathrm{~s}$ ) from the initial mass. We use the last time possible from the simulation as the final mass takes much of the total simulation time to reach its steady-state value. A least-squares regression gives a fit to our data of $1.3\left(R / R_{\odot}\right)^{-2.6} M_{\odot}$ for the $M_{\mathrm{ej}}=1.6 M_{\odot}$ data and $0.58\left(R / R_{\odot}\right)^{-2.2} M_{\odot}$ for the $M_{\mathrm{ej}}=2.6 M_{\odot}$ data. A variation of only 3 per cent in the values of lost mass is sufficient to obtain agreement between the fitted gradients, and therefore caution should be exercised in interpreting any difference between the two gradients. The dashed line in Figure 7 shows the prediction from TT98, the dotted line shows the fit obtained from Type Ia simulations compiled by Tauris (2015), and the dot-dashed green line is from Liu et al. (2015). The values of lost mass we find are comparable to those seen in Liu et al. (2015). Likewise, we find values of $\Delta M_{2}$ less than the values that are extrapolated from simulations of Type Ia supernovae, indicating

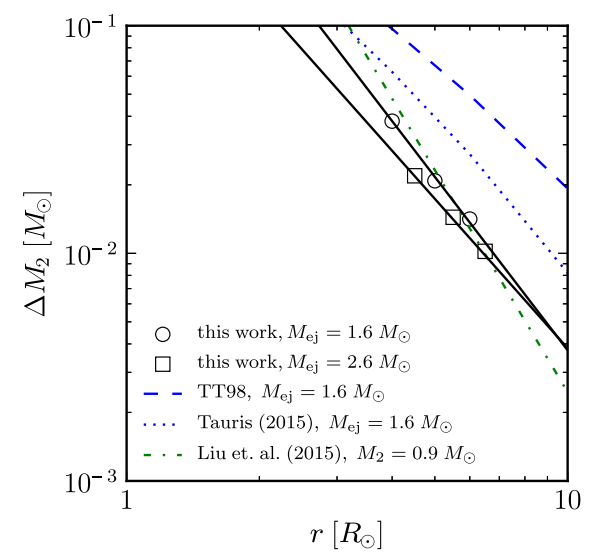

Figure 7 Final mass lost from the companion as a function of orbital separation. Circles show results with a primary star of $3 M_{\odot}$ and squares show results for a primary star of $4 M_{\odot}$. The secondary is $1 M_{\odot}$ in each case. The solid lines show the best fit power-laws for each ejecta mass. The comparison curves are from the theoretical predictions of WLK75 as adapted by TT98 and Tauris (2015) (rescaled to our initial conditions), as well as the simulation results of Liu et al. (2015) for a $0.9 M_{\odot}$ companion star. Note that the comparison with Liu et al. (2015) is not fully equivalent, as both the ejecta mass and companion mass (and therefore radius) are slightly different.

that these values should be revised for the conditions of Type Ibc supernovae considered here.

There are some differences between our initial conditions and those from the previous work shown in Figure 7. Compared with previous Type Ia simulations, our explosion energies and ejecta masses are both slightly different. Additionally, the companion radius, $R_{2}$, shrinks slightly after relaxation of the $\mathrm{SPH}$ models compared with the radius from the MESA model. The predictions in TT98 and Tauris (2015) depend on these quantities in particular within the geometric parameter $\Psi$, used originally by Wheeler et al. (1975), defined as

$$
\Psi=\left(\frac{R_{2}}{2 a}\right)^{2}\left(\frac{m_{\mathrm{shell}}}{m_{2}}\right)\left(\frac{v_{\mathrm{ej}}}{v_{\mathrm{esc}}}-1\right) .
$$

This parameter is used in the determination of $x_{\text {crit }}$ as well as $F_{\text {strip }}$ and $F_{\text {ablate }}$ in Wheeler et al. (1975) using tabulated data for an $n=3$ polytrope. For our comparisons, we adjust these quantities (and therefore $\Psi$ ) in the TT98 and Tauris (2015) estimates to match the initial conditions of our simulations. Furthermore, the simulations in Liu et al. (2015) also use a slightly different companion mass and ejecta mass, and so their results are not completely equivalent to ours. The structure of the companion star has been shown to substantially effect the magnitude of removed mass in simulations of Type Ia supernovae (Liu et al. 2012; Meng et al. 2007; Pan et al. 2012). Slight differences in companion models can therefore responsible for some of the discrepancies. 
3.3 Momentum transfer and the velocity of the companion When the orbital separation is very small, the impact of the ejecta causes not only a significant loss of mass from the companion star but also a large change in velocity. The largest change in velocity of the companion occurs during the transfer of momentum from the shell in the initial impact. However, as the end of the shell passes over the far side of the companion, there is an overpressure acting on this side of the star when the shock converges on this axis. This causes the companion to receive a slight change in momentum in the direction opposite to the shell motion (which has been suggested in other simulations such as Fryxell and Arnett 1981; Marietta et al. 2000). In the theory of TT98, $\mathbf{v}_{\text {im }}$ is defined to be an effective velocity that not only accounts for the momentum imparted to the companion by the passage of the shell but also the subsequent change in momentum due to (potentially asymmetric) mass loss.

We found that measuring the velocity of the companion with respect to the neutron star is complicated by the difficulty to define the baryonic centres of the binary system with the ejecta that had not yet left the binary system, the oscillatory behaviour of the companion star as a result of the shell impact, and the Brownian motion of the neutron star due to the shot-noise of the limited resolution in its vicinity.

As an alternative technique, we set up a co-rotating frame of reference that matches the original circular orbital motion. Up until the shell impact, there is no component of velocity of the companion perpendicular to this direction of motion. During and after the impact, the companion (as well as the mass unbound from it) gains a component of velocity, and therefore momentum, in the radial direction with respect to this frame. We use this to measure the momentum delivered to the companion and the material removed from the companion, as well as the radial impact velocities.

The left-hand panel of Figure 8 shows the component of momentum in the radial direction for material unbound from the companion that was not unbound in the previous time step. The first peak is due to the large amount of material initially stripped from the companion star by the shell impact. This figure also clearly indicates the burst of material out of the back of the star, seen as the second peak in the left-hand panel. The right-hand panel shows the breakdown of momenta in the radial direction for unbound and bound material originally from the companion (and their sum). This gives an alternative indication of $\eta$, where we see that although less than half of the total incident momentum in the shell is delivered to this material in total, only $\lesssim 30$ per cent of the momentum is delivered to the (bound material of the) companion star.

As the interaction of the shell with the companion is not instantaneous, we must define a point at which we measure the impact velocity. Up until the second peak seen in the left-hand panel of Figure 8, the radial velocity gained by the companion is dominated by the interaction with the passing shell. Once this interaction has ended, there is an additional, growing radial component in velocity from the eccentricity induced in the orbit. The change in gradient of $p_{\mathrm{r}}$ for the companion, seen in the right-hand panel of Figure 8, marks the end of the impact phase, which corresponds to just after the second peak in the left-hand panel. We define this to be the point at which we measure the impact velocity. Up to this point, the contribution to the radial velocity from any induced eccentricity is small.

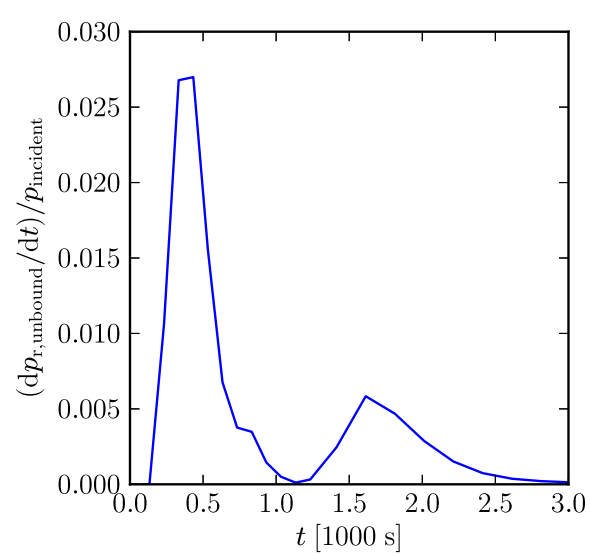

(a)

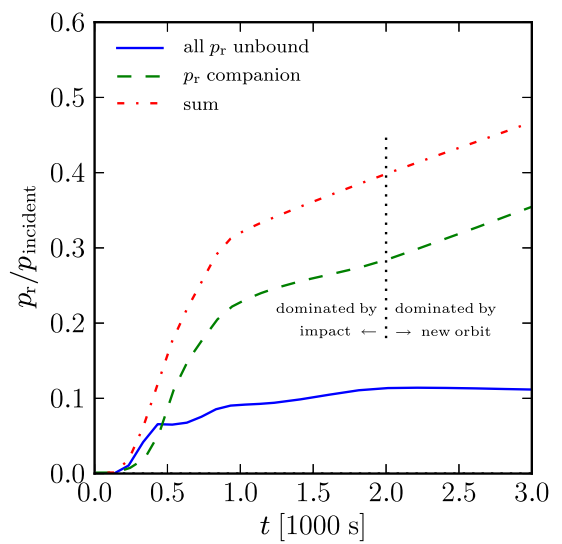

(b)

Figure 8 Components of momenta in the radial direction of (a) newly unbound material from the companion (material that was not unbound the previous snapshot) and (b) the total unbound mass from the companion, and bound mass in the companion, and the sum of these two values. Values are shown as a fraction of the total incident momentum calculated for the cross-section of shell material that impacts the companion. The example shown is for a $3 M_{\odot}$ primary and an orbital separation of $6 R_{\odot}$. 


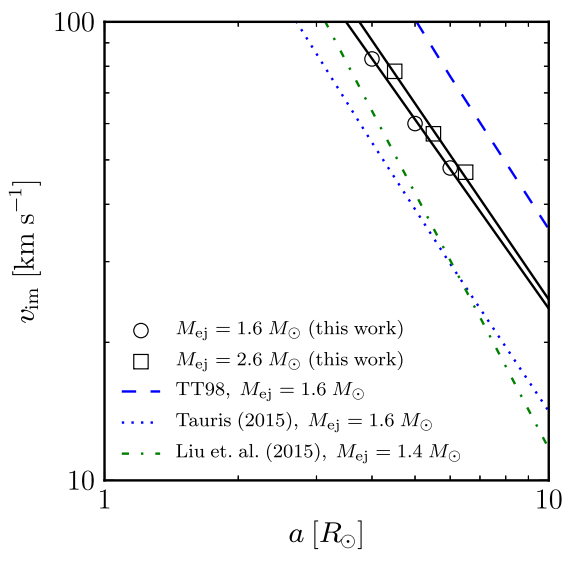

Figure 9 Magnitude of the impact velocity, $v_{i m}$, imparted to the companion star as a function of orbital separation $\boldsymbol{a}$. Markers and line styles correspond to those used in Figure 7. Again, as for Figure 7, the comparison with Liu et al. (2015) is not fully equivalent due to the slightly different ejecta mass and companion parameters.

Our final impact velocity magnitudes are shown in Figure 9. A least-squares regression gives a fit to these data of $556\left(R / R_{\odot}\right)^{-1.4} \mathrm{~km} \mathrm{~s}^{-1}$ for the $M_{\mathrm{ej}}=1.6 M_{\odot}$ data and $652\left(R / R_{\odot}\right)^{-1.4} \mathrm{~km} \mathrm{~s}^{-1}$ for the $M_{\mathrm{ej}}=2.6 M_{\odot}$ data. The velocities for both ejecta mass conditions follow a similar gradient to earlier work presented in TT98 and Tauris (2015), although it is not quite as steep as the -1.9 power-law of Liu et al. (2015). The overall scaling differs from previous work, however. The early estimate from TT98 of the impact velocities used a value of $\eta \approx 0.5$ (see Equation 4 ), whereas fits in Tauris (2015) and Liu et al. (2015) sit closer to $\eta \approx 0.2$. Our impact velocities lie in between these values, corresponding to $\eta \approx 0.3$. At the point of measurement of $v_{\mathrm{im}}$, there will already be a small contribution in the measured $v_{\text {im }}$ from the growing radial velocity component due to the eccentricity of the new orbit. However, even if we were to define the measured impact velocity to be earlier (before the shock convergence at the far side of the star), this still produces values of $v_{\mathrm{im}}$ that are larger than those seen in Liu et al. (2015). We consider a possible cause of differences in results in Section 3.3.1.

Finally, we also consider the effect of drag from the remaining material on the companion velocity, noting that there is still a non-negligible density of gas interior to the ejecta shell. For a conservative estimate of this drag force from the innermost ejecta, we neglect any outward velocity of this gas, and take a density of $10^{-3} \mathrm{~g} \mathrm{~cm}^{-3}$ in this material. With these values, the drag force on the companion will be

$$
F_{\mathrm{drag}}=\frac{1}{2} \rho v_{2}^{2} C_{\mathrm{drag}} A_{2} \approx 2 \times 10^{28} \mathrm{~N}
$$

for $v_{2}=300 \mathrm{~km} \mathrm{~s}^{-1}$, where we approximate the drag coefficient of the star with a solid sphere value of $C_{\mathrm{drag}} \approx 0.5$. For a companion mass of $M_{2}=1 M_{\odot}$ the acceleration associated with this drag is therefore only $10^{-5} \mathrm{~km} \mathrm{~s}^{-2}$. Although small, drag induced by the lower-velocity ejecta may appreciably alter the final velocity of the companion when integrated over a long timescale.

\subsubsection{Ejecta profiles}

We investigate in more detail our ejecta profiles as a potential cause of the discrepancy between our impact velocities and those of Liu et al. (2015). Previous work, such as that of Liu et al. (2015), has often initialised the ejecta with the assumption that it is in a homologous expansion by the time it impacts the companion, so that, for a given $t, v \propto R$. The density and velocity profiles in this ejecta are constructed from broken power-law fits to analytic treatments of the shock through the progenitor. These treatments have, in particular, been based on the polytropic envelopes (or onedimensional structure models) of supergiant stars, and the power-law fits are to the (small and large $R$ ) asymptotic limits of a varying density gradient in the ejecta (Chevalier and Soker 1989; Matzner and McKee 1999).

In Figure 10, we show the variation of ejecta density and velocity as a function of radius from the centre of mass (by averaging the SPH particles over concentric shells) and compare with an analytic function from the equations used in Liu et al. (2015). We also show, in the bottom panel of Figure 10, the distribution of velocity over mass in the ejecta. It is clear from Figure 10 that in the ejecta from our helium star models we have a shallower density gradient through much of the outer regions compared with the power-law profiles. In this outer ejecta, the velocity and density are also higher in our models. As the impact velocity has a strong dependence on this high-velocity ejecta (Liu et al. 2015), this can explain increased impact velocities seen in our simulations.

Finally, we examined the ejecta for large-scale asymmetries by determining the shell-averaged radial profiles of density and velocity in hemispheres corresponding to the directions toward and away from the companion star. We found that the values in either direction agreed to within a few per cent, and therefore do not produce a discernible difference on the logarithmic plots in Figure 10.

\subsection{Properties of the larger-scale SNR}

The amount of accretion on the companion has previously been shown to decrease with increasing shell velocity (Fryxell and Arnett 1981); therefore, the high ejecta velocities in Type Ibc supernovae lead us to expect little pollution of the companion with supernova material. Indeed, we find negligible pollution of the companion star. The converse - pollution of the SNR with material from the companion - can be appreciable. A few $10^{-2} M_{\odot}$ of hydrogen- 


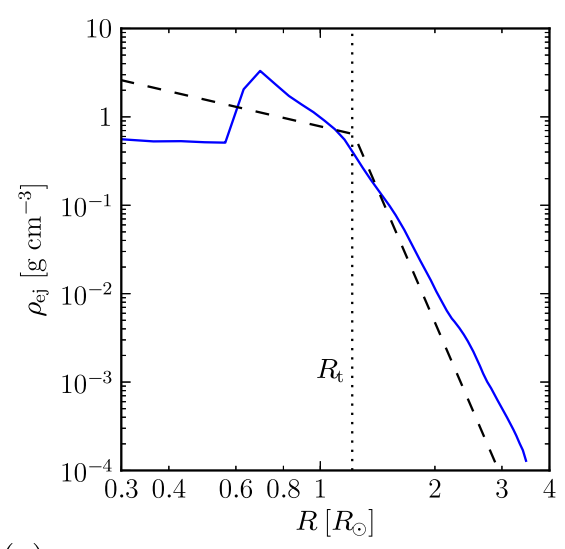

(a)

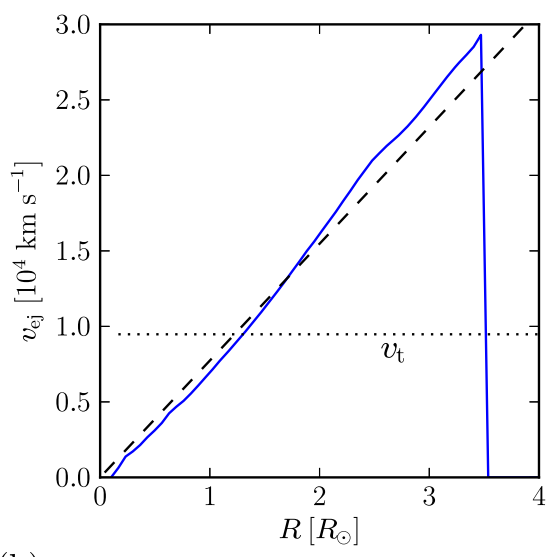

(b)

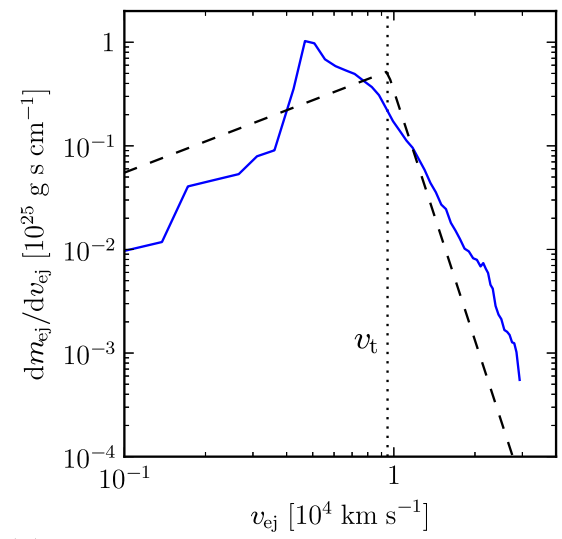

(c)

Figure 10 Blue lines show the profiles of (a) density, (b) magnitude of velocity and (c) the mass distribution of velocity within the ejecta for our simulations (binned in radial shells form the centre of mass of the ejecta). Black dashed lines show the power-law profiles used in Liu et al. (2015). Both cases were calculated for a time of $90 \mathrm{~s}$ after the supernova. The transition velocity (and radius at which this occurs) in the Liu et al. (2015) profiles are given as dotted lines.

rich material may be lost from the companion by the passing shell in our simulations, which may be detectable as an asymmetry in the metallicity of the SNR on the side of the companion.

Figure 11 shows a 3D rendering of the SNR and companion at $10^{3} \mathrm{~s}$ after the moment of the supernova. At this point, a hole has been created in the passing shell due to the presence of the companion, which is seen to persist at later times. The hole in the ejecta caused by the companion is approximately 30 degrees in size for the minimum orbital separations.

Even if an ejecta hole cannot be detected morphologically, the presence of a hole in SNR ejecta may allow the inference of a companion from a burst of radiation generated during the impact with the companion, which can escape through the less optically thick region of the companion shadow cone (Kasen 2010). The hole may persist to late stages of the SNR despite some amount of refilling due to the subsequent rarefaction wave along with hydrodynamic instabilities (García-Senz et al. 2012; Kasen 2010).

Not only do we observe a hole in the SNR due to the companion star, but we also see an increase in the density in a ring surrounding the hole, as shown most clearly in Figure 11. As shell material impacts the outer part of the companion star, where material is stripped and swept up with the ejecta, this ring of gas is also compressed in contrast with the freely expanding ejecta that do not interact with the companion. Aside from augmentation of the early supernova light curve, our results also suggest that ringlike enhancements in density of the SNR could indicate the presence of a companion star. Ring-like structures may be easier to detect than a hole in the SNR as the enhancement in density may also be associated with an increase in radiative losses in the ring.

As the full composition (the mass fraction of each species as determined by the MESA model) is recorded for 


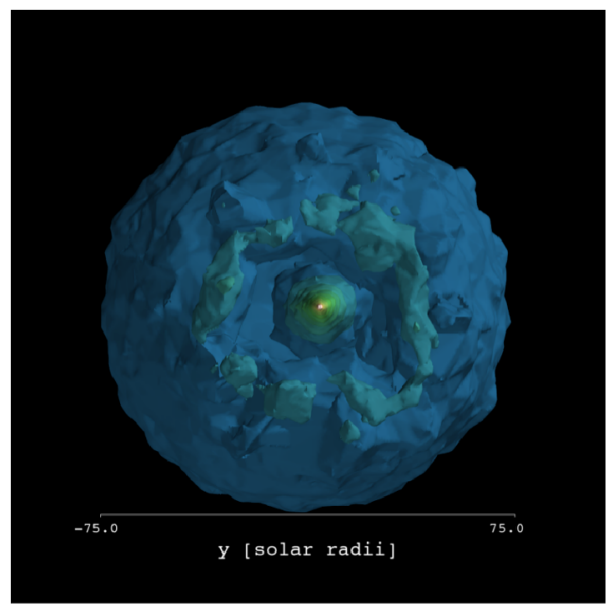

(a)

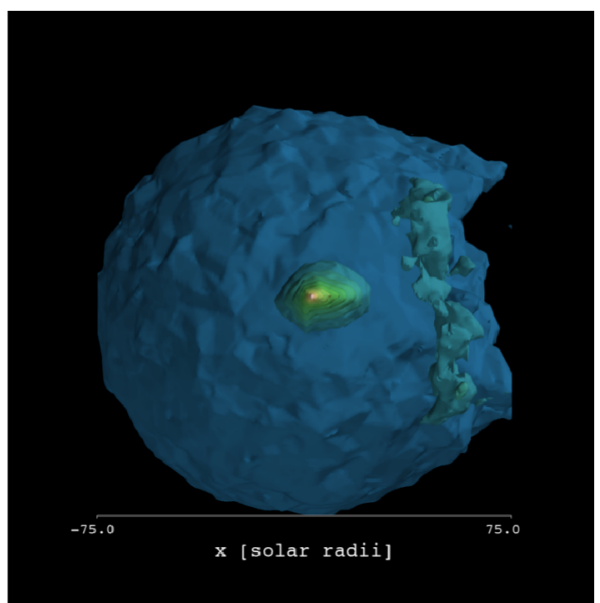

(b)

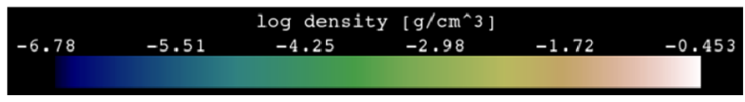

Figure $11 \mathrm{~A}$ 3D rendering of the gas density in the system $10^{3} \mathrm{~s}$ after the supernova, viewed down the $x$-axis (a; the original axis of the binary) and $y$-axis (b). The companion is still distorted due to the impact, and has produced a hole in the expanding ejecta. We used the software Mayavi2 (Ramachandran and Varoquaux 2011) for the visualisation.

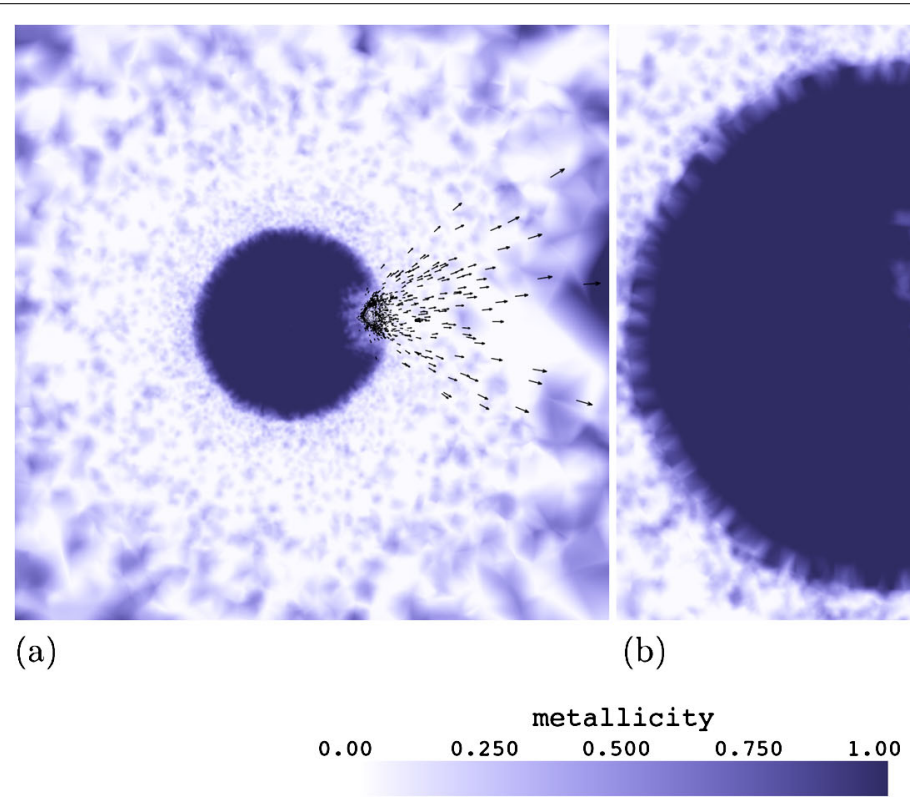

Figure $12 \mathrm{~A} 30 R_{\odot}$ by $30 R_{\odot}$ slice through the $x-y$ (initial orbital) plane showing the mean metallicity $\left(1-X_{\mathrm{H}}-X_{\mathrm{He}}\right)$ within $1 R_{\odot}$ of the plane at (a) $733 \mathrm{~s}$ and (b) $2028 \mathrm{~s}$ after a supernova in a $3 M_{\odot}$ primary at an orbital separation of $\mathbf{4} \boldsymbol{R}_{\odot}$. Black vectors show samples of the momentum of the material unbound from the companion star projected onto the orbital plane, as in Figure 5.

each SPH particle, we are able to trace the dispersion of this material from the progenitor in the subsequent SNR. We do not, however, calculate the changes in composition during the supernova itself; as much of this process involves transmutation of one metal species to another in the stellar interior (where the metallicity remains $Z \sim 1$ ), we therefore limit the present discussion to the overall metallicity of the material.

Figure 12 illustrates that the metallicity of the SNR is highest in the innermost regions, where the ejecta rep- 
resents material nearest the core of the supernova progenitor. A hole develops in this high-metallicity ejecta, at first primarily due to the shadow of the companion star (panel (a)). At later times (panel (b)), the ablation of companion material further reduces the metallicity of a large fraction of the inner part of SNR in the direction of the companion. The orbital motion of the companion star within the inner SNR during this longer period of ablation can also enlarge the region over which the gas is enriched with hydrogen.

\subsection{Post-impact evolution of the companion}

Following the stripping and ablation of mass from the outer layers of the companion star, we used AMUSE to investigate the stellar evolution of the companion and compare with an unperturbed stellar model evolving from the main-sequence. As we associate composition with each $\mathrm{SPH}$ particle from the original stellar model, we were able to convert the final SPH state of the companion back to a one-dimensional structure model by an inversion of the method to construct the SPH model outlined in Section 2.2. After the model is loaded back into MESA, we continue the stellar evolution and compared with an undisturbed companion model.

As we find negligible contamination of the companion with ejecta material, the difference in evolution is effectively due to the reduction in mass of the star. A $1 M_{\odot}$ star with metallicity $Z=0.02$ evolves to the through to a carbon-oxygen WD at $12.1 \mathrm{Gyr}$ in MESA. On the other hand, the $1 M_{\odot}$ model which has lost $0.04 M_{\odot}$ of material from the supernova impact reaches this stage at a later age of $14.0 \mathrm{Gyr}$. Although the final age of the stars is noticeably different, there is little evolutionary difference between the two models on an HR diagram. It may, therefore, be difficult to distinguish a companion that has lost part of its envelope due to a supernova from $T_{\text {eff }}$ and $L$ alone. Nevertheless, the stripping and contamination in the outer layers of the star still has the potential to produce differences in chemical abundances that are spectrally distinguishable from the coeval stellar population (see also, for example, Pan et al. 2012).

More immediately, after the impact of the shell on the companion, a large amount of thermal energy is deposited in the outer layers of the star, which will dramatically affect its appearance over approximately the thermal timescale of the outer layers. To investigate this in more detail, in Figure 13 we plot the density and internal energy of a companion star subjected to a supernova in a $3 M_{\odot}$ primary star at a separation of $4 R_{\odot}$.

For this example, the total excess internal energy, integrated over the spherical shell $\gtrsim 0.6 R_{\odot}$, is $\Delta U \approx 10^{47} \mathrm{erg}$. If this were instantaneously converted to radiation, the excess energy would be released on a diffusion timescale, which we calculate to be $\sim 10 \mathrm{yr}$ in these low-density outer layers of the star. ${ }^{\mathrm{g}}$

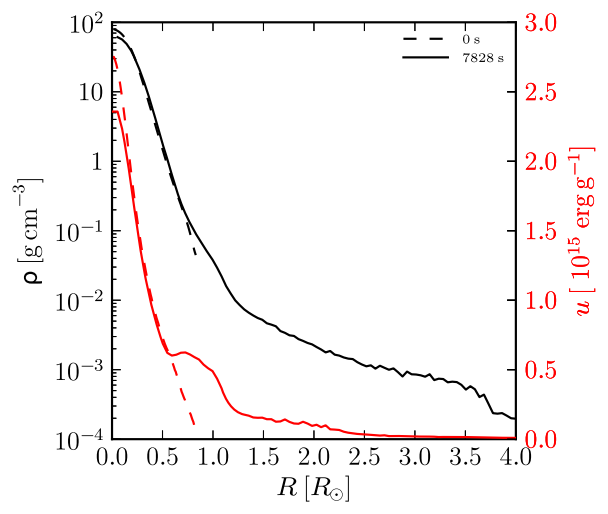

Figure 13 Density (left-hand axis) and internal energy (right-hand axis) of the companion star before (dashed lines) and $7828 \mathrm{~s}$ after (solid lines) the supernova explosion. These properties were determined by averaging across radial shells concentric with the centre of mass of the companion. All SPH particles bound to the companion were within a radius of $4.5 R_{\odot}$.

The thermal timescale of the diffuse outer layers of such an expanded star is shorter than the canonical solar thermal timescale, and is of the order $t_{\text {therm }} \approx 10^{3} \sim 10^{4} \mathrm{yr}$ (Marietta et al. 2000; Podsiadlowski 2003). Nevertheless, as the thermal timescale is still orders of magnitude larger than the diffusion timescale, we expect the luminosity due to this excess energy to be limited by the former.

A rough estimate of the luminosity can then be found from $\Delta U / t_{\text {therm }} \approx 10^{2} \sim 10^{3} L_{\odot}$. In practice, however, the luminosity will gradually decline from a peak value to the main-sequence luminosity ${ }^{\mathrm{h}}$ over roughly the thermal timescale (Podsiadlowski 2003).

Finally, we note that in the example of Figure 13, the furthest extent of the SPH particles in the companion was $\sim 4 R_{\odot}$. If the companion star were to continue expanding to a larger extent, its radius would encompass the neutron star in systems that remain bound, complicating the subsequent evolution of the system. This could increase the likelihood of accretion of material onto the neutron star, or even result in a merger between the neutron star and the companion. The interaction may also re-circularise the orbit after an eccentricity was gained from a kick to the neutron star during the supernova.

\section{Discussion and conclusions}

For supernovae in close binaries, the impact of the ejecta shell can have non-negligible effects on the mass and velocity of the companion star. The change in momentum of the companion is used in predictions of the final velocities of runaway stars from supernova-dissociated binaries, as seen in the recent work of Tauris (2015). These predictions are important for determining the level of contamination from these stars in searches for hypervelocity stars from 
other origins, such as the Hills mechanism with the supermassive black hole in the Galactic Centre (Hills 1988; Yu and Tremaine 2003).

We have performed SPH simulations of supernovae in close binaries to study the consequences of the shell impact on the companion. The overall hydrodynamic phenomena and trends we observe during these simulations are broadly consistent with previous studies of Type Ia (Liu et al. 2012; Marietta et al. 2000; Pakmor et al. 2008; Pan et al. 2012), Type Ibc (Liu et al. 2015) and Type II (Hirai et al. 2014) supernovae. In addition, we find that the gradient in the impact velocity predicted by Wheeler et al. (1975) matches our results well, with some modification of the $\eta$ parameter representing the total momentum received by the companion.

While this work was in preparation, Liu et al. (2015) presented work on the effect of a Type Ibc supernova shell impacting a companion star. As with Liu et al. (2015), we find that the magnitude of mass loss and impact velocity of the companion is less than early estimates. However, the velocity induced onto the companion due to the shell impact in their work is a factor of 1.5 2 lower than our results. Although it is not straightforward to separate the causes of such discrepancies, there are a number of differences between our calculations. One is the structure of the companion star, which is known to affect both the mass loss and impact velocity results (Liu et al. 2012, 2015; Meng et al. 2007; Pan et al. 2012). A more notable difference is that in our simulations the shell is naturally formed from the supernova explosion mechanism, as opposed to the introduction of the supernova ejecta by an analytic description. This results in a different ejecta structure, and more momentum carried in the leading edge of the ejecta, which is important in determining the final impact velocity (Liu et al. 2015).

Using the predictions from our simulations, we return to the question of runaway velocities of the components of supernova-disrupted binaries considered in TT98 and Tauris (2015). We have created a python code that calculates the final speeds derived by TT98 in order to investigate the analytic predictions with our simulation results. In this Monte Carlo code, an impulsive increase in velocity, $\mathbf{w}$, is imposed to the neutron star, randomly oriented from an isotropic distribution over a sphere. This is achieved by mapping from a uniform random distribution over $t \in(0,1]$ to $2 \pi t$ for the angle $\phi$, and from a uniform random distribution over $u \in[0,1]$ to $\cos ^{-1}(2 u-1)$ for the angle $\theta$. Figure 14 shows a comparison of the distributions of speeds with (red) and without (blue) the effect of applying $\mathbf{v}_{\text {im }}$ and mass loss in the companion star.

From Figure 14, it is evident that, although adding an impact velocity to the companion (perpendicular to its orbital velocity) increases the minimum companion speed, it also in fact reduces the maximum companion speed. To clarify the discrepancies in the distributions that occur when

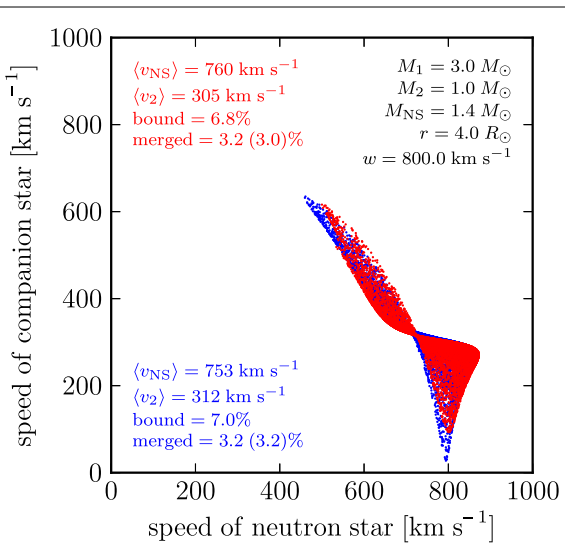

Figure 14 Comparison of Monte Carlo sampling over NS kick orientation for the case with no impact effects on the companion (blue) and with impact effects as determined from our simulations (red). Both cases show $10^{4}$ samples of NS kick orientation with a uniform on distribution over the unit sphere. The magnitude of the NS kick velocity, $w$, is fixed at $800 \mathrm{~km} \mathrm{~s}^{-1}$ throughout. Mean values of the runaway velocities, as well as percentages of cases where the binary components remain bound and merged (and, in parentheses, merged cases that were calculated as bound). Neither bound nor merged cases appear in these distributions.

adding $\mathbf{v}_{\text {im }}$, we consider the effect of NS kick angles on the final velocity of the companion star in disrupted binaries in Figure 15, analogous to Figure 4 in Tauris (2015). The white regions for high $\theta$ in each panel represent binaries that remain bound after the NS kick (and thus the runaway velocity is undefined). The grey regions represent NS kick angles for which the NS and companion star merge after the supernova. It can be seen from the lower panel that the effect of applying an impact velocity to the companion star can stabilise the systems where the NS kick is counter-aligned with the NS orbital velocity. In fact, the small region of parameter space giving large values of $v_{2}$ at $\phi=0$ and high $\theta$ is removed after adding $\mathbf{v}_{\text {im }}$ (due to these systems now remaining bound). This explains the potentially counterintuitive result that by adding an additional velocity to the companion star in fact reduces the maximum possible velocity of runaway stars.

The main results of our work are as follows:

- We follow the supernova from just after the core bounce in a helium star generated from a stellar evolution model. Exploding a supernova in such a model produces an ejecta profile that is different from that used in previous work, which has employed an analytic function for the ejecta distribution derived from the theory of shocks travelling through a one-dimensional atmosphere. The progenitor model used here is still somewhat artificial in construction, with a constant mass loss parameter late in its evolution. As understanding of Type Ibc progenitors 


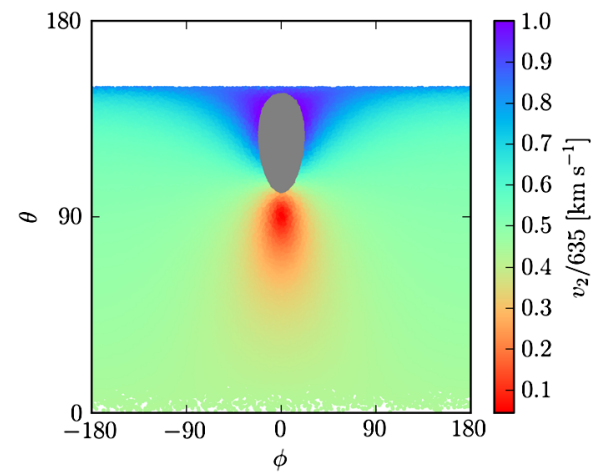

(a)

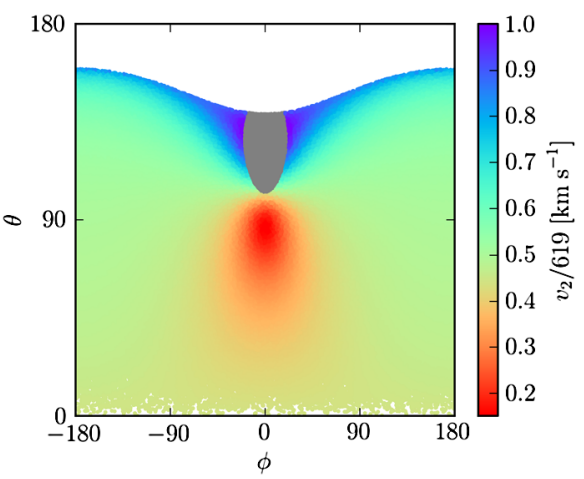

(b)

Figure 15 Distribution of NS kick angles from the $x$-axis (aligned with the pre-kick NS orbital vector) for the same parameters as the Monte Carlo runs shown in Figure 14 (but with $2 \times 10^{5}$ samples to better fill the space in angles). The span of $\theta$ over $\phi=0$ defines the orbital plane, where $\theta=0$ is for a kick aligned with the NS orbital velocity vector. Panel (a) shows the case of no impact effects on the companion (blue distribution in Figure 14) and panel (b) shows the case where impact effects determined from our simulations are included (red distribution in Figure 14). Colours represent the magnitude of the companion star from disrupted binaries (as a fraction of the maximum runaway velocity). Grey shows cases where the NS and companion star merge. The white regions for large $\theta$ are cases where the binary remains bound and so there is no runaway companion.

improves (for some recent investigations, see Kim et al. 2015), future work would benefit from a more realistic progenitor model by modelling the mass loss processes in detail.

- We have investigated the mass removed from the companion in the very close binary separations seen in Type Ibc supernovae, as well as the net change in momentum of the companion star due to the shell impact and later ablation of material. We show that an extrapolation of results from Type Ia supernova simulations do not provide a good fit to the Type Ibc scenarios considered here, and we provide updated fits to the distance-dependence of these results. In agreement with Liu et al. (2015), we find lower values of the removed mass and impact velocity of the companion compared to earlier estimates; however, we find generally larger impact velocities.

Discrepancies in the results are due not only to differences in companion models but also to differences in the distribution of momentum in the ejecta.

- We investigated the morphology of the SNR shortly after the shell has passed the companion, as well as the pollution of the SNR with material stripped from the companion, which, for the case of Type Ibc supernovae, may be a detectable fraction of the total mass in the ejecta (several $10^{-2} M_{\odot}$ out of $\sim 2 M_{\odot}$ ). The metallicity of the SNR is found to be highest in the inner regions of the SNR, and in this region the ablation of hydrogen from the outer layers of the companion star can dilute the metallicity on the side of the SNR facing the companion, resulting in a strong asymmetry in metallicity in the orbital plane.

- The companion star is additionally found to modify the morphology of the SNR in two distinct ways: as anticipated, a hole forms in the SNR on the side of the companion; also, an increase in the SNR density is seen in a ring around the hole, which may enhance the luminosity in SNR observations.

- We have also considered subsequent state of the companion after the shell impact and removal of mass during the shell impact, and have confirmed that the luminosity of the star can be orders of magnitude larger than the main-sequence luminosity during the release of thermal energy from the shock-heated outer layers.

\section{Competing interests}

The authors declare that they have no competing interests.

\section{Authors' contributions}

AR ran the simulations, analysed the data and wrote the draft of this paper. SPZ had the idea that initiated this work and assisted with the interpretation of the results. EMR assisted with the interpretation of the impact momenta and velocity results and the runaway star Monte Carlo results. All of the authors took part in discussions concerning the results and contributed corrections and improvements on the draft of the manuscript. All authors read and approved the final manuscript.

\section{Acknowledgements}

We are grateful to Nathan de Vries for his assistance with an early version of the supernova explosion code in AMUSE, and to Arjen van Elteren and Inti Pelupessy for AMUSE code development. We also thank the two anonymous referees, whose comments helped improve this manuscript. This work was supported by the Netherlands Research Council (NWO grant numbers 612.071.305 [LGM] and 639.073.803 [VICI]), the Netherlands Research School for Astronomy (NOVA), the Interuniversity Attraction Poles Programme initiated by the Belgian Science Policy Office (IAP P7/08 CHARM), and by the European 
Union's Horizon 2020 Research and Innovation programme under grant agreement No. 671564.

\section{Endnotes}

a More recently, the 'Bethe', $B$, has also been proposed as an equivalent unit in honour of Hans Bethe's work on supernovae (Weinberg 2006; Woosley and Heger 2007)

b As shown by Pan et al. (2012) for the Type la case, both orbital motion and rotation produce no substantial difference in the impact velocity gained by the companion. However, it is possible that a high rotation rate in the companion can help unbind a small additional amount of shock-heated material from the surface of the companion due to the additional rotational energy.

c www.amusecode.org

d This 'canonical' value of the solar metallicity may be an overestimate; see Asplund et al. (2009) for a review.

e Randomisation of the angular orientation of the particles has the undesirable effect of the additional shot noise it generates in the initial density distribution; however, the further step of damped relaxation used here will ultimately result in a glass-like configuration.

$f$ This smooths thermal energy discontinuities and is used in capturing the vortices seen in KHI. However, there has been some debate on the causes of $\mathrm{KH}$ s suppression in $\mathrm{SPH}$; see, for example, the discussion in Gabbasov et al. (2014).

9 To obtain this value, we integrated from $0.6 R_{\odot}$ to the surface of the star assuming a Thomson cross-section.

h In fact, the luminosity from nuclear energy generation can drop lower than the main-sequence value, due to the reduction in central temperature and pressure following the initial expansion (Marietta et al. 2000).

Received: 15 October 2015 Accepted: 18 February 2016 Published online: 01 March 2016

\section{References}

Asplund, M, Grevesse, N, Sauval, AJ, Scott, P: The chemical composition of the Sun. Annu. Rev. Astron. Astrophys. 47, 481-522 (2009). doi:10.1146/annurev.astro.46.060407.145222. arXiv:0909.0948

Bersten, MC, Benvenuto, OG, Folatelli, G, Nomoto, K, Kuncarayakti, H, Srivastav, S, Anupama, GC, Quimby, R, Sahu, DK: iPTF13bvn: the first evidence of a binary progenitor for a type lb supernova. Astron. J. 148, 68 (2014). doi:10.1088/0004-6256/148/4/68. arXiv:1403.7288

Bruenn, SW, Mezzacappa, A, Hix, WR, Lentz, EJ, Bronson Messer, OE, Lingerfelt, EJ, Blondin, JM, Endeve, E, Marronetti, P, Yakunin, KN: Axisymmetric ab initio core-collapse supernova simulations of 12-25 $M_{\text {Sun }}$ stars. Astrophys. J. 767, L6 (2013). doi:10.1088/2041-8205/767/1/L6. arXiv:1212.1747

Cao, Y, Kulkarni, SR, Howell, DA, Gal-Yam, A, Kasliwal, MM, Valenti, S, Johansson, J, Amanullah, R, Goobar, A, Sollerman, J, Taddia, F, Horesh, A, Sagiv, I, Cenko, SB, Nugent, PE, Arcavi, I, Surace, J, Woźniak, PR, Moody, DI, Rebbapragada, UD, Bue, BD, Gehrels, N: A strong ultraviolet pulse from a newborn type la supernova. Nature 521, 328-331 (2015). doi:10.1038/nature14440. arXiv:1505.05158

Chevalier, RA, Soker, N: Asymmetric envelope expansion of supernova 1987A. Astrophys. J. 341, 867-882 (1989). doi:10.1086/167545

Church, RP, Dischler, J, Davies, MB, Tout, CA, Adams, T, Beer, ME: Mass transfer in eccentric binaries: the new oil-on-water smoothed particle hydrodynamics technique. Mon. Not. R. Astron. Soc. 395, 1127-1134 (2009). doi:10.1111/j.1365-2966.2009.14619.x. arXiv:0902.3509

Colgate, SA: Ejection of companion objects by supernovae. Nature 225 247-248 (1970). doi:10.1038/225247a0

Couch, SM, O'Connor, EP: High-resolution three-dimensional simulations of core-collapse supernovae in multiple progenitors. Astrophys. J. 785, 123 (2014). doi:10.1088/0004-637X/785/2/123. arXiv:1310.5728

Couch, SM, Ott, CD: The role of turbulence in neutrino-driven core-collapse supernova explosions. Astrophys. J. 799, 5 (2015) doi:10.1088/0004-637X/799/1/5. arXiv:1408.1399

de Vries, N, Portegies Zwart, S, Figueira, J: The evolution of triples with a Roche lobe filling outer star. Mon. Not. R. Astron. Soc. 438, 1909-1921 (2014). doi:10.1093/mnras/stt1688. arXiv:1309.1475
Dessart, L, Blondin, S, Hillier, DJ, Khokhlov, A: Constraints on the explosion mechanism and progenitors of type la supernovae. Mon. Not. R. Astron. Soc. 441, 532-550 (2014). doi:10.1093/mnras/stu598. arXiv:1310.7747

Duquennoy, A, Mayor, M: Multiplicity among solar-type stars in the solar neighbourhood. II - Distribution of the orbital elements in an unbiased sample. Astron. Astrophys. 248, 485-524 (1991)

Eggleton, P: Evolutionary Processes in Binary and Multiple Stars. Cambridge University Press, Cambridge (2006)

Eggleton, PP: The evolution of low mass stars. Mon. Not. R. Astron. Soc. 151, 351 (1971)

Eggleton, PP: Approximations to the radii of Roche lobes. Astrophys. J. 268, 368 (1983). doi:10.1086/160960

Eldridge, JJ, Fraser, M, Maund, JR, Smartt, SJ: Possible binary progenitors for the type Ib supernova iPTF13bvn. Mon. Not. R. Astron. Soc. 446, 2689-2695 (2015). doi:10.1093/mnras/stu2197. arXiv:1408.4142

Fremling, C, Sollerman, J, Taddia, F, Ergon, M, Valenti, S, Arcavi, I, Ben-Ami, S, Cao, Y, Cenko, SB, Filippenko, AV, Gal-Yam, A, Howell, DA: The rise and fall of the type lb supernova iPTF13bvn. Not a massive Wolf-Rayet star. Astron. Astrophys. 565, A114 (2014). doi:10.1051/0004-6361/201423884. arXiv:1403.6708

Fryxell, BA, Arnett, WD: Hydrodynamic effects of a stellar explosion on a binary companion star. Astrophys. J. 243, 994-1002 (1981). doi:10.1086/158664

Gabbasov, R, Klapp-Escribano, J, Suárez-Cansino, J, Sigalotti, L: Numerical simulations of the Kelvin-Helmholtz instability with the Gadget-2 SPH code. In: Klapp, J, Medina, A (eds.) Experimental and Computational Fluid Mechanics. Environmental Science and Engineering, pp. 291-298. Springer, Berlin (2014). ISBN 978-3-319-00115-9. doi:10.1007/978-3-319-00116-6_24

García-Senz, D, Badenes, C, Serichol, N: Is there a hidden hole in type la supernova remnants? Astrophys. J. 745, 75 (2012). doi:10.1088/0004-637X/745/1/75. arXiv:1 110.4267

González Hernández, Jl, Ruiz-Lapuente, P, Tabernero, HM, Montes, D, Canal, R, Méndez, J, Bedin, LR: No surviving evolved companions of the progenitor of SN 1006. Nature 489, 533-536 (2012). doi:10.1038/nature11447. arXiv:1210.1948

Hanke, F, Müller, B, Wongwathanarat, A, Marek, A, Janka, H-T: SASI activity in three-dimensional neutrino-hydrodynamics simulations of supernova cores. Astrophys. J. 770, 66 (2013). doi:10.1088/0004-637X/770/1/66. arXiv:1303.6269

Hills, JG: Hyper-velocity and tidal stars from binaries disrupted by a massive Galactic black hole. Nature 331, 687-689 (1988). doi:10.1038/331687a0

Hirai, R, Sawai, H, Yamada, S: The outcome of supernovae in massive binaries; removed mass, and its separation dependence. Astrophys. J. 792, 66 (2014). doi:10.1088/0004-637X/792/1/66. arXiv:1404.4297

Hurley, JR, Pols, OR, Tout, CA: Comprehensive analytic formulae for stellar evolution as a function of mass and metallicity. Mon. Not. R. Astron. Soc. 315, 543-569 (2000). doi:10.1046/j.1365-8711.2000.03426.x. arXiv:astro-ph/0001295

Janka, H-T: Explosion mechanisms of core-collapse supernovae. Annu. Rev. Nucl. Part. Sci. 62, 407-451 (2012). doi:10.1146/annurev-nucl-102711-094901. arXiv:1206.2503

Kane, J, Drake, RP, Remington, BA: An evaluation of the Richtmyer-Meshkov instability in supernova remnant formation. Astrophys. J. 511, 335-340 (1999). doi:10.1086/306685

Kasen, D: Seeing the collision of a supernova with its companion star. Astrophys. J. 708, 1025-1031 (2010). doi:10.1088/0004-637X/708/2/1025. arXiv:0909.0275

Kerzendorf, WE, Yong, D, Schmidt, BP, Simon, JD, Jeffery, CS, Anderson, J, Podsiadlowski, P, Gal-Yam, A, Silverman, JM, Filippenko, AV, Nomoto, K, Murphy, SJ, Bessell, MS, Venn, KA, Foley, RJ: A high-resolution spectroscopic search for the remaining donor for Tycho's supernova. Astrophys. J. 774, 99 (2013). doi:10.1088/0004-637X/774/2/99. arXiv:1210.2713

Kim, H-J, Yoon, S-C, Koo, B-C: Observational properties of type lb/c supernova progenitors in binary systems. Astrophys. J. 809, 131 (2015). doi:10.1088/0004-637X/809/2/131. arXiv:1506.06354

Kuncarayakti, H, Maeda, K, Bersten, MC, Folatelli, G, Morrell, N, Hsiao, EY, González-Gaitán, S, Anderson, JP, Hamuy, M, de Jaeger, T, Gutiérrez, CP Kawabata, KS: Nebular phase observations of the type-lb supernova iPTF13bvn favour a binary progenitor. Astron. Astrophys. 579, A95 (2015). doi:10.1051/0004-6361/201425604 arXiv:1504.01473 
Kusenko, A, Segrè, G: Pulsar velocities and neutrino oscillations. Phys. Rev. Lett. 77, 4872-4875 (1996). doi:10.1103/PhysRevLett.77.4872. arXiv:hep-ph/9606428

Lai, D, Rasio, FA, Shapiro, SL: Collisions and close encounters between massive main-sequence stars. Astrophys. J. 412, 593-611 (1993). doi:10.1086/172946

Leonard, DC: Constraining the type la supernova progenitor: the search for hydrogen in nebular spectra. Astrophys. J. 670, 1275-1282 (2007). doi:10.1086/522367. arXiv:0710.3166

Li, W, Bloom, JS, Podsiadlowski, P, Miller, AA, Cenko, SB, Jha, SW, Sullivan, M, Howell, DA, Nugent, PE, Butler, NR, Ofek, EO, Kasliwal, MM, Richards, JW, Stockton, A, Shih, H-Y, Bildsten, L, Shara, MM, Bibby, J, Filippenko, AV, Ganeshalingam, M, Silverman, JM, Kulkarni, SR, Law, NM, Poznanski, D, Quimby, RM, McCully, C, Patel, B, Maguire, K, Shen, KJ: Exclusion of a luminous red giant as a companion star to the progenitor of supernova SN 2011 fe. Nature 480, 348-350 (2011). doi:10.1038/nature10646. arXiv:1109.1593

Liu, ZW, Pakmor, R, Röpke, FK, Edelmann, P, Wang, B, Kromer, M, Hillebrandt, W, Han, ZW: Three-dimensional simulations of the interaction between type la supernova ejecta and their main sequence companions. Astron. Astrophys. 548, A2 (2012). doi:10.1051/0004-6361/201219357. arXiv:1209.4458

Liu, Z-W, Tauris, TM, Roepke, FK, Moriya, TJ, Kruckow, M, Stancliffe, RJ, Izzard, RG: The interaction of core-collapse supernova ejecta with a companion star. ArXiv e-prints, arXiv:1509.03633 (2015)

Lopez, LA, Ramirez-Ruiz, E, Castro, D, Pearson, S: The Galactic supernova remnant W49B likely originates from a jet-driven, core-collapse explosion. Astrophys. J. 764, 50 (2013). doi:10.1088/0004-637X/764/1/50. arXiv:1301.0618

Lundqvist, P, Nyholm, A, Taddia, F, Sollerman, J, Johansson, J, Kozma, C, Lundqvist, N, Fransson, C, Garnavich, PM, Kromer, M, Shappee, BJ, Goobar, A: No trace of a single-degenerate companion in late spectra of supernovae $2011 \mathrm{fe}$ and 2014J. Astron. Astrophys. 577, A39 (2015). doi:10.1051/0004-6361/201525719. arXiv:1502.00589

Maoz, D, Mannucci, F, Nelemans, G: Observational clues to the progenitors of type la supernovae. Annu. Rev. Astron. Astrophys. 52, 107-170 (2014). doi:10.1146/annurev-astro-082812-141031. arXiv:1312.0628

Marietta, E, Burrows, A, Fryxell, B: Type IA supernova explosions in binary systems: the impact on the secondary star and its consequences. Astrophys. J. Suppl. Ser. 128, 615-650 (2000). doi:10.1086/313392. arXiv:astro-ph/9908116

Maruyama, T, Kajino, T, Yasutake, N, Cheoun, M-K, Ryu, C-Y: Asymmetric neutrino emission from magnetized proto-neutron star matter including hyperons in relativistic mean field theory. Phys. Rev. D 83(8), 081303 (2011). doi:10.1103/PhysRevD.83.081303. arXiv:1009.0976

Mattila, S, Lundqvist, P, Sollerman, J, Kozma, C, Baron, E, Fransson, C, Leibundgut, B, Nomoto, K: Early and late time VLT spectroscopy of SN $2001 \mathrm{el}$ - progenitor constraints for a type la supernova. Astron. Astrophys. 443, 649-662 (2005). doi:10.1051/0004-6361:20052731. arXiv:astro-ph/0501433

Matzner, CD, McKee, CF: The expulsion of stellar envelopes in core-collapse supernovae. Astrophys. J. 510, 379-403 (1999). doi:10.1086/306571 arXiv:astro-ph/9807046

Meng, X, Chen, X, Han, Z: The impact of type la supernova explosions on the companions in a binary system. Publ. Astron. Soc. Jpn. 59, 835-840 (2007). doi:10.1093/pasj/59.4.835

Milisavljevic, D, Soderberg, AM, Margutti, R, Drout, MR, Howie Marion, G, Sanders, NE, Hsiao, EY, Lunnan, R, Chornock, R, Fesen, RA, Parrent, JT, Levesque, EM, Berger, E: SN 2012au: a golden link between superluminous supernovae and their lower-luminosity counterparts. Astrophys. J. Lett 770, L38 (2013). doi:10.1088/2041-8205/770/2/L38. arXiv:1304.0095

Olling, RP, Mushotzky, R, Shaya, EJ, Rest, A, Garnavich, PM, Tucker, BE, Kasen, D, Margheim, S, Filippenko, AV: No signature of ejecta interaction with a stellar companion in three type la supernovae. Nature 521, 332-335 (2015). doi:10.1038/nature14455

Pakmor, R, Röpke, FK, Weiss, A, Hillebrandt, W: The impact of type la supernovae on main sequence binary companions. Astron. Astrophys. 489, 943-951 (2008). doi:10.1051/0004-6361:200810456. arXiv:0807.3331

Pakmor, R, Edelmann, P, Röpke, FK, Hillebrandt, W: Stellar GADGET: a smoothed particle hydrodynamics code for stellar astrophysics and its application to type la supernovae from white dwarf mergers. Mon. Not. R. Astron. Soc. 424, 2222-2231 (2012). doi:10.1111/j.1365-2966.2012.21383.x. arXiv:1205.5806
Pan, K-C, Ricker, PM, Taam, RE: Impact of type la supernova ejecta on binary companions in the single-degenerate scenario. Astrophys. J. 750, 151 (2012). doi:10.1088/0004-637X/750/2/151. arXiv:1203.1932

Paxton, B, Bildsten, L, Dotter, A, Herwig, F, Lesaffre, P, Timmes, F: Modules for experiments in stellar astrophysics (MESA). Astrophys. J. Suppl. Ser. 192, 3 (2011). doi:10.1088/0067-0049/192/1/3. arXiv:1009.1622

Pelupessy, Fl, van Elteren, A, de Vries, N, McMillan, SLW, Drost, N, Portegies Zwart, SF: The astrophysical multipurpose software environment. Astron. Astrophys. 557, A84 (2013). doi:10.1051/0004-6361/201321252. arXiv:1307.3016

Podsiadlowski, P: On the evolution and appearance of a surviving companion after a type la supernova explosion. Mon. Not. R. Astron. Soc. submitted. arXiv:astro-ph/0303660 (2003)

Portegies Zwart, S, McMillan, S, Harfst, S, Groen, D, Fujii, M, Nualláin, BÓ, Glebbeek, E, Heggie, D, Lombardi, J, Hut, P, Angelou, V, Banerjee, S, Belkus, H, Fragos, T, Fregeau, J, Gaburov, E, Izzard, R, Jurić, M, Justham, S, Sottoriva, A, Teuben, $\mathrm{P}$, van Bever, J, Yaron, O, Zemp, M: A multiphysics and multiscale software environment for modeling astrophysical systems. New Astron. 14, 369-378 (2009). doi:10.1016/j.newast.2008.10.006. arXiv:0807.1996

Portegies Zwart, S, McMillan, SLW, van Elteren, E, Pelupessy, I, de Vries, N: Multi-physics simulations using a hierarchical interchangeable software interface. Comput. Phys. Commun. 183, 456-468 (2013). doi:10.1016/j.cpc.2012.09.024. arXiv:1204.5522

Ramachandran, P, Varoquaux, G: Mayavi: 3D visualization of scientific data. Comput. Sci. Eng. 13(2), 40-51 (2011)

Rasio, FA, Lombardi, JC Jr.: Smoothed particle hydrodynamics calculations of stellar interactions. J. Comput. Appl. Math. 109, 213-230 (1999). arXiv:astro-ph/9805089

Rastegaev, DA: Multiplicity and period distribution of population II field stars in solar vicinity. Astron. J. 140, 2013-2024 (2010) doi:10.1088/0004-6256/140/6/2013. arXiv:1009.4596

Rimoldi, A, Rossi, EM, Piran, T, Portegies Zwart, S: The fate of supernova remnants near quiescent supermassive black holes. Mon. Not. R. Astron. Soc. 447, 3096-3114 (2015). doi:10.1093/mnras/stu2630. arXiv:1501.02819

Ruiz-Lapuente, P, Comeron, F, Méndez, J, Canal, R, Smartt, SJ, Filippenko, AV, Kurucz, RL, Chornock, R, Foley, RJ, Stanishev, V, Ibata, R: The binary progenitor of Tycho Brahe's 1572 supernova. Nature 431, 1069-1072 (2004). doi:10.1038/nature03006. arXiv:astro-ph/0410673

Sana, H, de Mink, SE, de Koter, A, Langer, N, Evans, CJ, Gieles, M, Gosset, E, Izzard, RG, Le Bouquin, J-B, Schneider, FRN: Binary interaction dominates the evolution of massive stars. Science 337, 444 (2012). doi:10.1126/science.1223344. arXiv:1207.6397

Schaefer, BE, Pagnotta, A: An absence of ex-companion stars in the type la supernova remnant SNR 0509-67.5. Nature 481, 164-166 (2012). doi:10.1038/nature10692

Scheck, L, Plewa, T, Janka, H-T, Kifonidis, K, Müller, E: Pulsar recoil by large-scale anisotropies in supernova explosions. Phys. Rev. Lett. 92(1), 011103 (2004). doi:10.1103/PhysRevLett.92.011103. arXiv:astro-ph/0307352

Scheck, L, Kifonidis, K, Janka, H-T, Müller, E: Multidimensional supernova simulations with approximative neutrino transport. I. Neutron star kicks and the anisotropy of neutrino-driven explosions in two spatial dimensions. Astron. Astrophys. 457, 963-986 (2006). doi:10.1051/0004-6361:20064855. arXiv:astro-ph/0601302

Smartt, SJ: Progenitors of core-collapse supernovae. Annu. Rev. Astron. Astrophys. 47, 63-106 (2009). doi:10.1146/annurev-astro-082708-101737. arXiv:0908.0700

Springel, V: The cosmological simulation code GADGET-2. Mon. Not. R. Astron. Soc. 364, 1105-1134 (2005). doi:10.1111/j.1365-2966.2005.09655.x. arXiv:astro-ph/0505010

Suwa, Y, Yoshida, T, Shibata, M, Umeda, H, Takahashi, K: Neutrino-driven explosions of ultra-stripped type Ic supernovae generating binary neutron stars. ArXiv e-prints, arXiv:1506.08827 (2015)

Tauris, TM: Maximum speed of hypervelocity stars ejected from binaries. Mon Not. R. Astron. Soc. 448, L6-L10 (2015). doi:10.1093/mnrasl/slu189. arXiv:1412.0657

Tauris, TM, Takens, RJ: Runaway velocities of stellar components originating from disrupted binaries via asymmetric supernova explosions. Astron. Astrophys. 330, 1047-1059 (1998)

Warren, DC, Blondin, JM: Three-dimensional numerical investigations of the morphology of type la SNRs. Mon. Not. R. Astron. Soc. 429, 3099-3113 (2013). doi:10.1093/mnras/sts566. arXiv:1210.7790

Weinberg, S: A Bethe unit. Phys. World 19(2), 17 (2006) 
Wheeler, JC, Lecar, M, McKee, CF: Supernovae in binary systems. Astrophys. J. 200, 145-157 (1975). doi:10.1086/153771

Wongwathanarat, A, Janka, H-T, Müller, E: Three-dimensional neutrino-driven supernovae: neutron star kicks, spins, and asymmetric ejection of nucleosynthesis products. Astron. Astrophys. 552, A126 (2013). doi:10.1051/0004-6361/201220636. arXiv:1210.8148

Woosley, SE, Heger, A: Nucleosynthesis and remnants in massive stars of solar metallicity. Phys. Rep. 442(1-6), 269-283 (2007).

doi:10.1016/j.physrep.2007.02.009. The Hans Bethe Centennial Volume 1906-2006

Xue, Z, Schaefer, BE: Newly determined explosion center of Tycho's supernova and the implications for proposed ex-companion stars of the progenitor. Astrophys. J. 809, 183 (2015). doi:10.1088/0004-637X/809/2/183. arXiv:1507.06347

Young, PA, Fryer, CL: Uncertainties in supernova yields. I. One-dimensional explosions. Astrophys. J. 664, 1033-1044 (2007). doi:10.1086/518081. arXiv:astro-ph/0612698

Yu, Q, Tremaine, S: Ejection of hypervelocity stars by the (binary) black hole in the Galactic Center. Astrophys. J. 599, 1129-1138 (2003). doi:10.1086/379546. arXiv:astro-ph/0309084

Zahn, J-P: Tidal friction in close binary stars. Astron. Astrophys. 57, 383-394 (1977)

\section{Submit your manuscript to a SpringerOpen ${ }^{\odot}$} journal and benefit from:

- Convenient online submission

- Rigorous peer review

- Immediate publication on acceptance

- Open access: articles freely available online

- High visibility within the field

- Retaining the copyright to your article

Submit your next manuscript at $\boldsymbol{~ s p r i n g e r o p e n . c o m ~}$ 